\title{
Potential role of tedizolid phosphate in the treatment of acute bacterial skin infections
}

This article was published in the following Dove Press journal:

Drug Design, Development and Therapy

2 April 2013

Number of times this article has been viewed

\section{Olatz Urbina' \\ Olivia Ferrández' \\ Mercè Espona' \\ Esther Salas' \\ Irene Ferrández ${ }^{2}$ \\ Santiago Grau'}

'Services of Hospital Pharmacy, Hospital Universitari del Mar, Universitat Autònoma de Barcelona, ${ }^{2}$ Ciència i Tecnologia dels Aliments, Pharmacy Department, Universitat de Barcelona, Barcelona, Spain
Correspondence: Olivia Ferrández Services of Hospital Pharmacy, Hospital Universitari del Mar, Passeig Marítim 25-29, E-08003 Barcelona, Spain

Tel +34 932483704

Fax+34932483256

Email94007@hospitaldelmar.cat
Abstract: Tedizolid phosphate (TR-701), a prodrug of tedizolid (TR-700), is a next-generation oxazolidinone that has shown favorable results in the treatment of acute bacterial skin and skin-structure infections in its first Phase III clinical trial. Tedizolid has high bioavailability, penetration, and tissue distribution when administered orally or intravenously. The activity of tedizolid was greater than linezolid against strains of Staphylococcus spp., Streptococcus spp., and Enterococcus spp. in vitro studies, including strains resistant to linezolid and those not susceptible to vancomycin or daptomycin. Its pharmacokinetic characteristics allow for a once-daily administration that leads to a more predictable efficacy and safety profile than those of linezolid. No hematological adverse effects have been reported associated with tedizolid when used at the therapeutic dose of $200 \mathrm{mg}$ in Phase I, II, or III clinical trials of up to 3 weeks of tedizolid administration. Given that the clinical and microbiological efficacy are similar for the 200, 300, and $400 \mathrm{mg}$ doses, the lowest effective dose of $200 \mathrm{mg}$ once daily for 6 days was selected for Phase III studies in acute bacterial skin and skin-structure infections, providing a safe dosing regimen with low potential for development of myelosuppression. Unlike linezolid, tedizolid does not inhibit monoamine oxidase in vivo, therefore interactions with adrenergic, dopaminergic, and serotonergic drugs are not to be expected. In conclusion, tedizolid is a novel antibiotic with potent activity against Gram-positive microorganisms responsible for skin and soft tissue infections, including strains resistant to vancomycin, linezolid, and daptomycin, thus answers a growing therapeutic need

Keywords: oxazolidinone, TR-700, TR-701 FA, tedizolid, skin and soft tissue infections, linezolid resistance

\section{Introduction}

Skin and soft tissue infections are one of the main reasons people seek health care and account for approximately 7\%-10\% of US hospital admissions. ${ }^{1,2}$ During the 2000-2004 period there was a $28.9 \%$ increase in the number of hospitalizations due to skin and soft tissue infections. ${ }^{3}$ A study conducted in Taiwan reported that approximately $7.7 \%$ of hospitalized patients presented with at least one skin and soft tissue infection during the 2005-2007 period. $^{4}$

Despite the fact that the etiology can be variable, Staphylococcus aureus is the microorganism most often involved. ${ }^{5}$ In recent years, an increase has been observed in the number of methicillin-resistant $S$. aureus (MRSA) strains, which makes the management of these infections more difficult. Thus, the rate of methicillin resistance in $S$. aureus strains from skin and soft tissue infections ranges from $22.8 \%$ in Europe to $35.9 \%$ in North America. ${ }^{5}$ 
Previously, infections due to MRSA strains were limited to the hospital setting. However, in the last decade, there has been an emergence of community-associated MRSA strains. For instance, a $59 \%$ prevalence of MRSA strains has been reported (range: $15 \%-74 \%$ ) in patients with skin and soft tissue infections who were admitted to emergency wards in eleven US cities. ${ }^{6}$ The USA 300 strain was identified in $97 \%$ of MRSA strains. Its high capacity for diffusion means that the USA 300 strain is now considered to be epidemic in the USA and is becoming significant in other parts of the world. ${ }^{7}$ Moreover, a decrease in this strain's sensitivity to several antibiotics has been observed, which could make the success of antibiotic treatment even more difficult. ${ }^{7}$ New clones of community MRSA have been reported to be on the rise in other European countries. ${ }^{8,9}$

Currently, the emergence of community-associated and hospital-origin MRSA strains from patients with skin and soft tissue infections seems to be under control. ${ }^{10}$ According to a recent study, the rate of skin and soft tissue infections due to community MRSA ranged from 76.8 (75.0-78.6) per 100,000 people/year in 2005 to $64.0(62.4-65.6)$ per 100,000 people/year in $2010(P=0.62) .{ }^{10}$ Thus, $60 \%$ of skin and soft tissue infections were caused by MRSA strains in 2005 , with an increase of up to $62 \%$ in 2006 and a significant annual reduction reaching $52 \%$ in 2010 . In addition, the rate of hospital MRSA infections decreased from 0.7 $(0.5-0.8)$ per 100,000 people/year in 2005 to $0.4(0.2-0.5)$ per 100,000 people/year in $2010(P=0.02)$. No trend was observed in the percentage of infections due to MRSA strains when the origin of the infections was the hospital $(P=0.96)$.

Vancomycin is the antibiotic that has been used most often to treat infections due to MRSA strains. However, its efficacy has been compromised by the emergence of $S$. aureus strains with heteroresistance to glycopeptide (heterogeneous vancomycin-intermediate $S$. aureus [hVISA] strains), intermediate resistance (vancomycin-intermediate S. aureus [VISA] strains) and, occasionally, complete resistance (vancomycin-resistant $S$. aureus strains). ${ }^{11-14}$

Linezolid was the first representative member of the oxazolidinone family introduced into the pharmaceutical market. Although it continues to show excellent activity, in recent years, MRSA strains have been isolated that are resistant to this antibiotic (minimum inhibitory concentration $[\mathrm{MIC}]>8 \mathrm{mcg} / \mathrm{mL}$ ) due to the mutations in $23 \mathrm{~S}$ ribosomal (r) RNA (namely G2576T) or ribosomal protein L3 or by the presence of the $c f r$ gene. ${ }^{15,16}$ The presence of the $c f r$ gene confers additional resistance to a high number of protein synthesis inhibitors (phenicols, lincosamides, streptogramins, pleuromutilins, oxazolidinones, and 16-membered macrolides $)^{17-19}$ and it has the capacity for horizontal transfer via mobile genetic elements..$^{20,21}$

Other antibiotics have been incorporated into the available therapeutic arsenal to compensate for the emergence of resistant strains, although we consider their use is not without limitations.

A close correlation has been described between the reduction in sensitivity to daptomycin and resistance to vancomycin in VISA and hVISA strains. ${ }^{22,23}$ This finding irequires precaution when considering daptomycin as a therapeutic alternative in infection due to VISA or hVISA strains. In terms of its safety profile, several severe cases of daptomycin-induced eosinophilic pneumonia ${ }^{24}$ have been described and treatment requires monitoring for possible muscular toxicity associated with the antibiotic. In particular, patients with impaired renal function or on concomitant treatment with statins should be monitored more closely. ${ }^{25}$

As a result of these issues and given the limited number of therapeutic options available for these infections, new therapeutic alternatives are being and have been developed for the treatment of skin and soft tissue infections, especially those produced by resistant Gram-positive microorganisms. One such new development is tedizolid (TR-700), which belongs to the family of oxazolidinones and is the active moiety of tedizolid phosphate (TR-701). One of its greatest advantages is that its activity is greater than that of vancomycin and linezolid against microorganisms involved mainly in skin and soft tissue infections, including strains of $S$. aureus that carry the $c f r$ gene, which, as mentioned, confers resistance to linezolid. ${ }^{20,26}$ Another of its advantages, which it shares with linezolid, is its availability both for parenteral and oral administration, allowing for outpatient treatment.

This review will describe the role of tedizolid phosphate (TR-701) in the treatment of skin and soft tissue infections.

\section{Pharmacology}

Tedizolid (TR-700 or DA-7157; formerly torezolid) is a next-generation oxazolidinone whose chemical differences compared with first-generation oxazolidinones grant it greater potency, a better spectrum of activity, and a lower resistance profile.

In addition to other advantages that will be listed further on in this paper, the prodrug tedizolid phosphate (TR-701 or DA-7218 or DA-7158; formerly torezolid phosphate) shares with linezolid the ability to be administered orally and intravenously; good bioavailability, tissue penetration, and distribution; and good activity against MRSA. ${ }^{27}$ 
Tedizolid phosphate is a basic compound with a molecular weight of 494.28 and chemical structure (R)-[3-(4-(2-(2methyl tetrazole-5-yl)pyridin-5-yl)-3-fluorophenyl)-2-oxo5-oxazolidinyl] methyl disodium phosphate, which is hydrolyzed by phosphatases to the active moiety tedizolid (R)-3-(4-(2-(2-methyl tetrazole-5 yl)pyridin-5-yl)-3 fluorophenyl)-5-hydroxy methyl oxazolidine-2-one. ${ }^{28}$

\section{Mechanism of action}

In a similar fashion to linezolid, tedizolid inhibits the synthesis of bacterial proteins by binding to the 50S subunit of the ribosome and preventing the formation of the "70S complex" formed by the binding of $\mathrm{N}$-formylmethionine-tRNA, the $50 \mathrm{~S}$ subunit, and the 70S subunit. In this way, it prevents the translation process in the first step of protein synthesis. ${ }^{29,30}$

\section{Pharmacokinetics}

\section{Absorption, distribution, metabolism, and excretion}

A study evaluated the pharmacokinetics in a murine model of tedizolid phosphate administered intravenously at doses of 5,10 and $20 \mathrm{mg} / \mathrm{kg}$ and orally at doses of 20,50 , and $100 \mathrm{mg} / \mathrm{kg}$, and of tedizolid $10 \mathrm{mg} / \mathrm{kg}$ administered both intravenously and orally. ${ }^{28}$ Dose-proportional pharmacokinetics were observed for all the doses evaluated. The oral administration of tedizolid phosphate resulted in rapid gastrointestinal absorption, with tedizolid detected in plasma within 15 minutes (time to maximum concentration $\left[\mathrm{T}_{\max }\right]$ : 25.5-65.0 minutes). Plasma area-under-the-curve (AUC) values for tedizolid were proportional to the doses of tedizolid phosphate administered. Similarly, the formation of tedizolid from tedizolid phosphate intravenous (IV) was rapid: it was detected in plasma at the first blood sampling time (1 minute) and rapidly reached $\mathrm{T}_{\max }$ (7.85-12.1 minutes) for the three doses studied. The AUC values for tedizolid detected in blood were proportional to the IV doses of tedizolid phosphate. Both tedizolid phosphate (oral and IV) and tedizolid were practically undetectable in urine after 24 hours and in the gastrointestinal tract of the animals, including feces. Another study analyzed the pharmacokinetics of tedizolid phosphate after the administration of a dose of $10 \mathrm{mg} / \mathrm{kg}$ in rats administered by both oral and IV routes. ${ }^{31}$ Table 1 shows the pharmacokinetic parameters of tedizolid phosphate and tedizolid after the administration of tedizolid phosphate to rats corresponding to these two studies. ${ }^{28,31}$

The pharmacokinetics of tedizolid phosphate in other species, such as rats and dogs has also been investigated. ${ }^{32}$ As in the two studies already discussed, tedizolid phosphate administered orally and intravenously was rapidly transformed into tedizolid both in vivo and in vitro in all the species analyzed.

In healthy humans, a bioavailability of $91.47 \%$ has been estimated after the oral administration of a dose of $200 \mathrm{mg}$ of tedizolid phosphate. ${ }^{33}$ Although one study found an increase in the $\mathrm{T}_{\max }$ value and reduction in the maximum concentration $\left(\mathrm{C}_{\max }\right)$ value of tedizolid after 10 hours of fasting, the AUC value was similar both with fasting and when administered with food. Thus, since AUC is the most important predictor

Table I Pharmacokinetic parameters of tedizolid phosphate and tedizolid after the administration of tedizolid phosphate to rats ${ }^{28,31}$

\begin{tabular}{|c|c|c|c|c|c|c|c|c|}
\hline \multicolumn{5}{|c|}{ Tedizolid phosphate IV } & \multicolumn{4}{|c|}{ Tedizolid phosphate OR } \\
\hline Dose (mg/kg) & $\begin{array}{l}5 \mathrm{mg} / \mathrm{kg} \\
(\mathrm{n}=7)^{28}\end{array}$ & $\begin{array}{l}10 \mathrm{mg} / \mathrm{kg} \\
(\mathrm{n}=7)^{28}\end{array}$ & $\begin{array}{l}20 \mathrm{mg} / \mathrm{kg} \\
(\mathrm{n}=9)^{28}\end{array}$ & $10 \mathrm{mg} / \mathrm{kg}^{31}$ & $\begin{array}{l}20 \mathrm{mg} / \mathrm{kg} \\
(\mathrm{n}=10)^{28}\end{array}$ & $\begin{array}{l}50 \mathrm{mg} / \mathrm{kg} \\
(\mathrm{n}=8)^{28}\end{array}$ & $\begin{array}{l}100 \mathrm{mg} / \mathrm{kg} \\
(\mathrm{n}=9)^{28}\end{array}$ & $10 \mathrm{mg} / \mathrm{kg}^{31}$ \\
\hline $\begin{array}{l}\text { AUC } \\
(\mathrm{mcg} * \mathrm{~min} / \mathrm{mL})\end{array}$ & $313 \pm 75.4$ & $44.7 \pm 16.9$ & $86.3 \pm 27.9$ & 3.216 & $44.7 \pm 16.9$ & $86.3 \pm 27.9$ & $163 \pm 40.0$ & 2.988 \\
\hline$T_{1 / 2}(\min )$ & $13.8 \pm 3.64$ & $133 \pm 30.8$ & $159 \pm 32.1$ & 205.2 & $133 \pm 30.8$ & $159 \pm 32.1$ & $162 \pm 29.6$ & 229.2 \\
\hline $\mathrm{C}_{\max }(\mathrm{mcg} / \mathrm{mL})$ & - & - & - & 8.81 & $0.214 \pm 0.0833$ & $0.320 \pm 0.102$ & $0.440 \pm 0.0995$ & 8.37 \\
\hline $\mathrm{T}_{\max }(\min )$ & - & - & - & - & $45.0 \pm 24.5$ & $80.6 \pm 26.5$ & $130 \pm 73.5$ & 30 \\
\hline $\mathrm{Cl}(\mathrm{mL} / \mathrm{min} / \mathrm{kg})$ & $16.0 \pm 3.40$ & $45.0 \pm 24.5$ & $80.6 \pm 26.5$ & 3.11 & - & - & - & - \\
\hline $\mathrm{Vd}_{\mathrm{ss}}(\mathrm{mL} / \mathrm{kg})$ & $84.7 \pm 14.2$ & $0.214 \pm 0.0833$ & $0.320 \pm 0.102$ & 0.918 & - & - & - & - \\
\hline$F(\%)$ & - & - & - & - & 3.44 & 2.66 & 2.51 & 92.8 \\
\hline Tedizolid IV & & & & & Tedizolid OR & & & \\
\hline $\begin{array}{l}\text { AUC } \\
(\mathrm{mcg} * \mathrm{~min} / \mathrm{mL})\end{array}$ & $905 \pm 105$ & $1.780 \pm 334$ & $4.070 \pm 1.140$ & & $2.890 \pm 857$ & $8.580 \pm 3.230$ & $17.500 \pm 6.710$ & \\
\hline$T_{1 / 2}(\min )$ & $106 \pm 12.0$ & $112+19.2$ & $115 \pm 29.8$ & & $158 \pm 24.5$ & $276 \pm 113$ & $366 \pm 85.7$ & \\
\hline $\mathrm{C}_{\max }(\mathrm{mcg} / \mathrm{mL})$ & $12.5 \pm 1.16$ & $20.8 \pm 3.41$ & $45.4 \pm 8.95$ & & $14.0 \pm 4.43$ & $22.9 \pm 8.13$ & $34.6 \pm 10.1$ & \\
\hline $\mathrm{T}_{\max }(\min )$ & $12.1 \pm 4.88$ & $7.85 \pm 4.88$ & $10.6 \pm 12.6$ & & $25.5 \pm 14.2$ & $43.1 \pm 24.6$ & $65.0 \pm 72.3$ & \\
\hline
\end{tabular}

Abbreviations: AUC, area under the curve; $\mathrm{Cl}$, total body clearance; $\mathrm{C}_{\max }$, maximum concentration; $\mathrm{F}$, absolute oral bioavailability; IV, intravenous; $\mathrm{OR}$, oral; $\mathrm{T}_{1 / 2}$, half-life; $\mathrm{T}_{\text {max }}$, time to maximum concentration; $\mathrm{Vd}_{\mathrm{ss}}$, volume of distribution at steady state. 
of efficacy, ${ }^{34}$ there are no dietary restrictions for taking the drug. ${ }^{35}$

Similar to the animal models, ${ }^{32}$ tedizolid phosphate administered orally or intravenously is rapidly transformed into tedizolid through hydrolysis of the phosphate group by phosphatases..$^{27}$ In addition, tedizolid was the major moiety detected ( $94.54 \%-98.23 \%$ of radioactivity administered) in plasma in a study that evaluated the oral administration of a single dose of radioactively labeled tedizolid phosphate in healthy adults. ${ }^{36}$

Two studies have evaluated the pharmacokinetics of tedizolid after the administration of multiple doses of tedizolid phosphate in healthy adults (Table 2). ${ }^{33,37}$ In one of them linezolid was used as a comparator treatment. ${ }^{37}$

The pharmacokinetic parameters of tedizolid have been determined in other studies in which increasing single doses of tedizolid phosphate were administered, both orally $(200-1200 \mathrm{mg})^{38}$ and intravenously $(100-400 \mathrm{mg}) .^{33}$ Less variability was observed in the systemic exposure of tedizolid when it was compared with linezolid. ${ }^{37}$ Thus, the pharmacokinetic parameters of tedizolid corresponding to day 1 were predictive of those observed at steady state. ${ }^{33,37}$ As in the animal models, the AUC and $\mathrm{C}_{\max }$ values of tedizolid showed linear pharmacokinetics almost proportional to the tedizolid phosphate dose, both in the studies with multiple doses and with single doses. ${ }^{37,38}$

Two studies have evaluated a model that reflects the superior pharmacokinetics of tedizolid phosphate. ${ }^{39,40}$ In a Phase II dose-ranging study of efficacy and tolerability of 200,300, and $400 \mathrm{mg}$ of tedizolid phosphate in the treatment of complicated skin and skin-structure infections, clinical cure and microbiological eradication rates were above $90 \%$ with all three doses. ${ }^{40}$ Population pharmacokinetics favored a two-compartment model over one disposition compartment. In the other Phase II study, nonlinear mixed effects modeling was performed to analyze pharmacokinetics of tedizolid phosphate in patients with acute bacterial skin and skinstructure infections (ABSSSI). ${ }^{39}$ Results showed that the pharmacokinetics was well described by a two-compartment model with zero-order dose delivery to the depot compartment and subsequent first-order absorption.

Plasma protein binding in healthy adults has been estimated between $86.1 \%$ and $91.9 \%$, with a mean of $89.44 \% \pm 1.58 \%{ }^{41}$

The volume of distribution of tedizolid has been reported to be between two and three times higher than the value observed with linezolid. ${ }^{37}$ Further, in a study with healthy adults, it was observed that the levels of free tedizolid in muscle and adipose tissue were similar to those observed

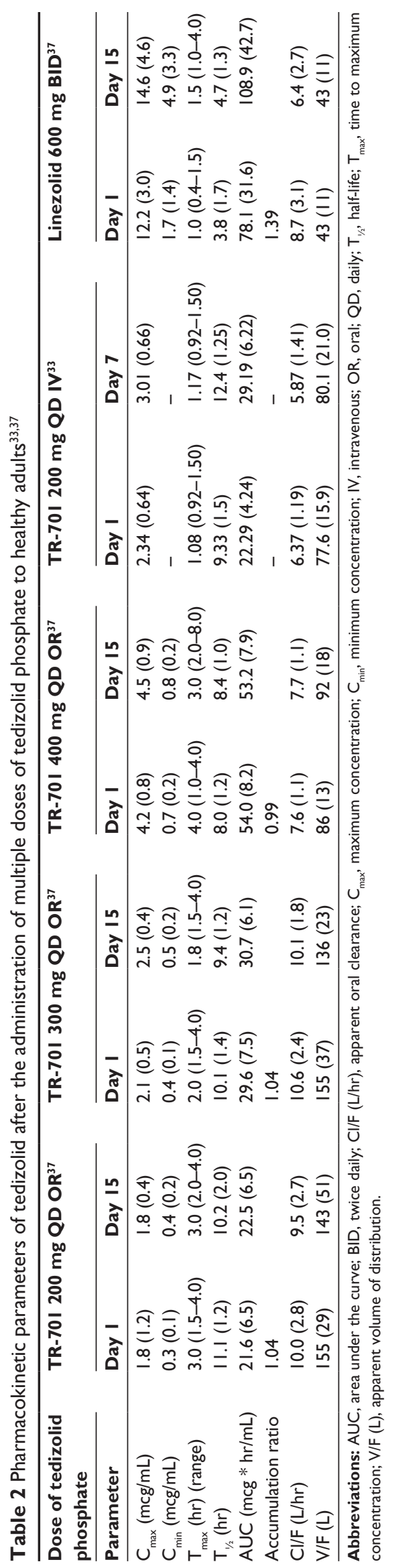


in plasma after the oral administration of a single dose of $600 \mathrm{mg}$ of tedizolid phosphate. ${ }^{42}$ Ratio of unbound AUC in tissues over unbound (free) AUC in plasma (fAUC tissue/ fAUC plasma) of tedizolid was $1.1 \pm 0.2$ in adipose tissue and $1.2 \pm 0.2$ in muscle tissue. These values, together with the rest of the pharmacokinetic parameters, support the administration of tedizolid phosphate in a single daily dose. ${ }^{33,37,38}$ Moreover, only modest accumulation of $\sim 30 \%$ tedizolid was observed in the studies that evaluated the administration of multiple doses of tedizolid phosphate, which would justify its safety profile..$^{33,43}$

Tedizolid is mostly eliminated in the feces; the urine is a minor elimination route. Two studies in healthy adults showed that less than $1 \%$ of the tedizolid phosphate dose administered was eliminated in urine unchanged or as tedizolid. ${ }^{37,38}$ Similarly, in a study in which a single dose of radioactively labeled tedizolid phosphate was administered to healthy adults, only $18 \%$ of the radioactive dose was recovered from the urine, whereas $81.5 \%$ was recovered from the feces. ${ }^{36}$ In both cases, the greatest metabolite was the sulfate analog, $10 \%$ or which was present in the urine and $69 \%$ in the feces. ${ }^{36} \mathrm{~N}$-demethylation and oxidation were minor elimination routes.

Three-stage hierarchical population pharmacokinetic modeling yielded the following estimations: geometric mean clearance of $8.28 \mathrm{~L} /$ hour (between-patient variability, 32.3\%), a volume of the central compartment of $71.4 \mathrm{~L}(24.0 \%)$, and a volume of the peripheral compartment of 27.9L (liters) $(35.7 \%)$ in patients with skin and soft tissue infections. ${ }^{40}$

\section{Special populations}

As mentioned, tedizolid phosphate is eliminated via the urine to a lesser extent and its pharmacokinetics is unchanged in subjects with severe renal impairment. Therefore, it is not necessary to adjust this drug in patients with reduced renal function. ${ }^{44}$ Likewise, the pharmacokinetics of tedizolid phosphate was not significantly altered in subjects with moderate to severe hepatic impairment, ${ }^{45}$ so dose adjustments should not be necessary in this patient population either.

In a recently completed Phase I clinical trial that compared tedizolid pharmacokinetics following oral administration of $200 \mathrm{mg}$ tedizolid phosphate in young patients (18-45 years) with elderly patients ( $>65$ years), no changes were observed..$^{46}$ In another study, the pharmacokinetic profile of a single oral or IV administration of $200 \mathrm{mg}$ tedizolid phosphate to healthy adolescents (12-17 years) was similar to that observed in adults. ${ }^{47}$

\section{Pharmacodynamics}

The pharmacodynamic characteristics of tedizolid phosphate were evaluated in a study comparing the efficacy of the dose fractionation of tedizolid phosphate in a neutropenic mousethigh model of methicillin-sensitive $S$. aureus (MSSA) and MRSA infections. ${ }^{34}$ The total tedizolid phosphate doses were provided as doses equivalent to tedizolid. The $\mathrm{AUC} / \mathrm{MIC}_{0-24 \mathrm{~h}}$, $\mathrm{T}>\mathrm{MIC}_{0-24 \mathrm{~h}}$, and $\mathrm{C}_{\max } / \mathrm{MIC}_{0-24 \mathrm{~h}}$ ratios of free drug were calculated for daily doses of tedizolid phosphate of 10, 20, 36 , and $72 \mathrm{mg} / \mathrm{kg} / 24$ hours, fractionated into one, two, or four times daily. According to the results, the AUC/MIC ${ }_{0-24 \mathrm{~h}}$ pharmacodynamic ratio obtained the best correlation with the efficacy of tedizolid $\left(\mathrm{r}^{2}: 0.984\right)$. The value of this ratio was $45(22.5 / 0.5)$ for a tedizolid phosphate regimen of $200 \mathrm{mg}$ daily on day 15 of treatment in healthy volunteers, which was higher than the value observed with linezolid $(108.9 / 4: 27.3){ }^{37}$

\section{Spectrum of activity}

In general, tedizolid shows excellent activity against aerobic Gram-positive microorganisms, including linezolid-resistant strains. In contrast, its use in Gram-negative infections is limited, given the high MIC of the antibiotic against these microorganisms.

Although the clinical breakpoints for tedizolid have not yet been defined, the objective of one study was to provide some conservative disc diffusion and MIC cut-off values for tedizolid (Table 3). ${ }^{48}$ In general, an MIC of $\leq 2 \mathrm{mcg} / \mathrm{mL}$ was used as the cut-off point of sensitivity.

\section{Aerobic Gram-positive microorganisms}

The activity of tedizolid in vitro has been found to be greater than that of linezolid against strains of Staphylococcus spp., Streptococcus spp., and Enterococcus spp., including strains resistant to linezolid and strains not susceptible to vancomycin or daptomycin (Table 4)..$^{31,48-55}$

In a Spanish study, it was observed that the minimum inhibitory concentration required to inhibit the growth of $50 \%$ of organisms $\left(\mathrm{MIC}_{50}\right)$ and minimum inhibitory concentration required to inhibit the growth of $90 \%$ of organisms $\left(\mathrm{MIC}_{90}\right)$ of tedizolid, linezolid, daptomycin, and vancomycin against MRSA strains from blood cultures were 0.25 and $0.50 \mathrm{mcg} /$ $\mathrm{mL}, 2$ and $4 \mathrm{mcg} / \mathrm{mL}, 0.5$ and $0.5 \mathrm{mcg} / \mathrm{mL}$, and 1 and $2 \mathrm{mcg} /$ $\mathrm{mL}$, respectively. ${ }^{49}$ In another study, the MIC of tedizolid ranged from $0.12 \mathrm{mcg} / \mathrm{mL}$ to $0.50 \mathrm{mcg} / \mathrm{mL}\left(\mathrm{MIC}_{90}\right.$ of $\left.0.25 \mathrm{mcg} / \mathrm{mL}\right)$ against $S$. aureus strains from skin and soft tissue infections. ${ }^{40}$ Similar results were reported in another study in $S$. aureus strains from skin and soft tissue infections, in which the $\mathrm{MIC}_{50}$ 
Table 3 Proposed minimum inhibitory concentration (MIC) cut-off point and disc diffusions for tedizolid ${ }^{48}$

\begin{tabular}{lll}
\hline Microorganism & Cut-off points $(\mathbf{S})$ & Disc diffusion using a disc of 20 or $\mathbf{1 0} \mathbf{~ m c g}$ \\
\cline { 2 - 3 } & MIC $(\mathbf{m c g} / \mathbf{m L})$ & $\geq 18$ \\
\hline Staphylococcus aureus & $\leq 2$ & $\geq 18$ \\
Coagulase-negative staphylococci & $\leq 2$ & $\geq 15 \mathrm{~mm}$ \\
Enterococcus spp., Streptococcus pneumoniae, & $\leq 2$ & No range recommended due to the reduced \\
and Streptococcus spp. other than S. pneumoniae & & number of strains tested \\
Corynebacterium jeikeium & $\leq 2$ & $\geq 15 \mathrm{~mm}$ \\
Listeria monocytogenes & $\leq 2$ &
\end{tabular}

Abbreviation: S, sensitive.

and $\mathrm{MIC}_{90}$ of tedizolid were $0.25 \mathrm{mg} / \mathrm{L}$, regardless of whether the isolated strain showed resistance to methicillin. ${ }^{55}$ These values were four- to eightfold lower than those observed with linezolid ( $\mathrm{MIC}_{50}$ and $\mathrm{MIC}_{90}$ of 1 and $2 \mathrm{mg} / \mathrm{L}$, respectively).

Similarly, tedizolid has shown excellent in vitro activity against 28 clinical strains of penicillin-resistant Streptococcus pneumoniae (MIC of penicillin $\mathrm{G} \geq 2 \mathrm{mcg} / \mathrm{mL}$ ), with $\mathrm{MIC}_{50}$ and $\mathrm{MIC}_{90}$ values of $0.25 \mathrm{mcg} / \mathrm{mL} .{ }^{56}$ These values were lower than those observed with linezolid $\left(\mathrm{MIC}_{50}\right.$ and $\mathrm{MIC}_{90}$ of $0.5 \mathrm{mcg} / \mathrm{mL}$ and $1.0 \mathrm{mcg} / \mathrm{mL}$, respectively). In another study, the MIC of tedizolid did not exceed the value of 0.25 and $0.12 \mathrm{mcg} / \mathrm{mL}$ against strains of Streptococcus agalactiae and Streptococcus pyogenes, respectively, from skin and soft tissue infections. ${ }^{40}$

Lastly, the $\mathrm{MIC}_{90}$ of tedizolid was between two and four times lower than that observed with linezolid against strains of enterococci $\left(\mathrm{MIC}_{90}\right.$ of $1.0 \mathrm{mcg} / \mathrm{mL}$ and $\mathrm{MIC}_{90}$ of $0.5 \mathrm{mcg} /$ $\mathrm{mL}$ against strains of enterococci sensitive and resistant to vancomycin, respectively). ${ }^{50}$

\section{Strains resistant to linezolid (non-characterized resistance mechanisms)}

Tedizolid has shown a high potency against strains of Grampositive microorganisms resistant to linezolid (Table 4). ${ }^{48,49,51-53}$ The MIC of tedizolid was $\leq 4 \mathrm{mcg} / \mathrm{mL}, \leq 8 \mathrm{mcg} / \mathrm{mL}$, and $\leq 16 \mathrm{mcg} / \mathrm{mL}$ in $88 \%, 96 \%$ and $>99 \%$ of 120 strains not sensitive to linezolid (72 strains of Enterococcus spp. and Streptococcus spp., and 48 strains of Staphylococcus spp.), respectively. ${ }^{53}$

An $\mathrm{MIC}_{90}$ of $2 \mathrm{mcg} / \mathrm{mL}$ for tedizolid has been reported against strains of $S$. aureus resistant to linezolid. ${ }^{51}$ In another study, the MIC of tedizolid was $0.5 \mathrm{mcg} / \mathrm{mL}$ against seven strains of MRSA (MIC against linezolid of $16 \mathrm{mcg} / \mathrm{mL}$ ) and between 0.25 and $4.00 \mathrm{mcg} / \mathrm{mL}$ against five strains of coagulase-negative staphylococci from blood culture (MIC range of linezolid: $16-256 \mathrm{mcg} / \mathrm{mL}$ ). ${ }^{49}$ Similar results have been obtained in other studies. ${ }^{51,52}$ In one of them, tedizolid showed an activity between eight and 16 times greater than linezolid against strains of Staphylococcus spp. resistant to linezolid, of which five strains of $S$. aureus also proved to be resistant to vancomycin and another five strains of the same species were not sensitive to daptomycin. ${ }^{51}$ Similarly, tedizolid has shown an activity eight times greater than linezolid against strains of Enterococcus spp. resistant to linezolid. ${ }^{51}$

Despite the fact that tedizolid shows greater activity than vancomycin against strains of Gram-positive microorganisms resistant to linezolid, ${ }^{53,54}$ the activity of glycopeptide has been shown to be greater in vitro against 40 strains of coagulase-negative staphylococci and one strain of Streptococcus oralis (MIC of vancomycin 1-2 $\mathrm{mcg} / \mathrm{mL}$ vs MIC of tedizolid 1 to $>32 \mathrm{mcg} / \mathrm{mL}$, MIC of vancomycin $0.5 \mathrm{mcg} / \mathrm{mL}$ vs MIC of tedizolid $2 \mathrm{mcg} / \mathrm{mL}$, respectively). ${ }^{53}$

\section{Strains with characterized linezolid-resistance mechanisms}

Tedizolid has maintained its activity against Gram-positive microorganisms with characterized resistance mechanisms against linezolid (Table 5). ${ }^{51}$ Thus, tedizolid's potency has been observed to be between eight and 16 times greater than that of linezolid in strains of $S$. aureus that carry the G2576U ${ }^{51}$ mutation (MIC of tedizolid $2-8 \mathrm{mcg} / \mathrm{mL}$ vs MIC of linezolid $16-64 \mathrm{mcg} / \mathrm{mL}$ ), and approximately 16 times greater in strains of $S$. aureus which carry the $c f r$ gene (MIC of tedizolid $0.5-1.0 \mathrm{mcg} / \mathrm{mL}$ vs MIC of linezolid $8-32 \mathrm{mcg} / \mathrm{mL}$ ).

Another study reported MIC values for tedizolid of between 0.5 and $16.0 \mathrm{mcg} / \mathrm{mL}$ when it was tested against 39 strains of Staphylococcus spp. carrying the G2576U mutation (MIC of linezolid 8 to $>32 \mathrm{mcg} / \mathrm{mL}$ ) and between 0.5 and $8.0 \mathrm{mcg} / \mathrm{mL}$ when tested against four strains of Staphylococcus spp. carrying the $c f r$ gene (MIC of linezolid 8 to $>32 \mathrm{mcg} / \mathrm{mL}) .^{53}$ Similarly, the MIC of tedizolid was 
Table 4 Activity of tedizolid (TR-700) and other antibiotics against Gram-positive microorganisms

\begin{tabular}{|c|c|c|c|c|c|}
\hline Microorganism & Strains tested (n) & Antibiotic & $\mathrm{MIC}_{50}(\mathrm{mcg} / \mathrm{mL})$ & $\mathrm{MIC}_{90}(\mathrm{mcg} / \mathrm{mL})$ & Range $(\mathrm{mcg} / \mathrm{mL})$ \\
\hline \multirow[t]{4}{*}{ Corynebacterium jeikeium } & $12^{48}$ & Tedizolid & 0.25 & 0.5 & $0.25-0.50$ \\
\hline & & Cefotaxime & 32 & 32 & $8-32$ \\
\hline & & Levofloxacin & $>16$ & $>16$ & $16>16$ \\
\hline & & Linezolid & 1 & I & $0.5-1.0$ \\
\hline Enterococcus faecalis & $54^{48}+73^{50}+49^{54}$ & TR-700 $48,50,54$ & $0.25-0.50$ & $0.5-1.0$ & $0.12-1.0$ \\
\hline \multirow[t]{8}{*}{ S to vancomycin } & & Linezolid ${ }^{48,50,54}$ & 2 & 2 & $0.5-4.0$ \\
\hline & & Vancomycin $^{50,54}$ & $\mathrm{I}-2$ & 2 & $0.5-4.0$ \\
\hline & & Cefotaxime $^{48}$ & $>64$ & $>64$ & $0.25>64.0$ \\
\hline & & Levofloxacin ${ }^{48,54}$ & $\mathrm{I}-2$ & $>16-64$ & $0.5-64.0$ \\
\hline & & Ampicillin ${ }^{54}$ & I & 4 & $0.25-8.0$ \\
\hline & & Erythromycin $^{54}$ & 4 & $>128$ & 0.12 to $>128.0$ \\
\hline & & Tetracycline $\mathrm{e}^{54}$ & 64 & 64 & $0.5-128.0$ \\
\hline & & Teicoplanin ${ }^{54}$ & 0.25 & 0.5 & $\leq 0.12-0.50$ \\
\hline \multirow[t]{9}{*}{ E. faecalis $\mathrm{R}$ to vancomycin } & $45^{48}+49^{50}+12^{54}$ & TR-700 $48,50,54$ & $0.25-0.5$ & 0.5 & $0.25-1.0$ \\
\hline & & Linezolid ${ }^{48,50,54}$ & $1-2$ & $1-2$ & $0.5-4.0$ \\
\hline & & Vancomycin ${ }^{50,54}$ & $>32$ to $>128$ & $>32$ to $>128$ & 4 to $>128$ \\
\hline & & Cefotaxime ${ }^{48}$ & $>64$ & $>64$ & 0.25 to $>64.0$ \\
\hline & & Levofloxacin ${ }^{48,54}$ & $>16-64$ & $>16-64$ & $0.5-128.0$ \\
\hline & & Ampicillin ${ }^{54}$ & 2 & 4 & $\mathrm{I}-4$ \\
\hline & & Erythromycin ${ }^{54}$ & $>128$ & $>128$ & $>128$ \\
\hline & & Tetracycline $e^{54}$ & 32 & 64 & $0.5-64.0$ \\
\hline & & Teicoplanin ${ }^{54}$ & 64 & 64 & $32-128$ \\
\hline \multirow[t]{3}{*}{ E. faecalis R to linezolid } & $16^{51}+12^{53}$ & TR-700 51,53 & $2-4$ & 4 & $0.5-8.0$ \\
\hline & & Linezolid ${ }^{51}$ & 32 & 32 & $8-32$ \\
\hline & & Vancomycin ${ }^{51}$ & 1 & $>16$ & I to $>16$ \\
\hline Enterococcus faecium & $52^{48}+53^{50}+30^{54}$ & TR-700 $48,50,54$ & $0.25-0.5$ & $0.25-1$ & $0.06-2.00$ \\
\hline \multirow[t]{8}{*}{$S$ to vancomycin } & & Linezolid ${ }^{48,50,54}$ & 2 & $2-4$ & $0.5-4.0$ \\
\hline & & Vancomycin ${ }^{50,54}$ & $0.5-1.0$ & $0.5-1.0$ & $0.25-4.00$ \\
\hline & & Cefotaxime ${ }^{48}$ & $>64$ & $>64$ & 0.5 to $>64$ \\
\hline & & Levofloxacin ${ }^{48,54}$ & $4-64$ & $>16-64$ & $0.5-128.0$ \\
\hline & & Ampicillin ${ }^{54}$ & $>128$ & $>128$ & I to $>128$ \\
\hline & & Erythromycin ${ }^{54}$ & $>128$ & $>128$ & 0.25 to $>128.00$ \\
\hline & & Tetracycline $e^{54}$ & 0.5 & 1 & $0.12-32.00$ \\
\hline & & Teicoplanin ${ }^{54}$ & 0.5 & 0.5 & $0.25-2.00$ \\
\hline \multirow[t]{9}{*}{ E. faecium $\mathrm{R}$ to vancomycin } & $52^{48}+51^{50}+29^{54}$ & TR-700 $48,50,54$ & $0.12-0.5$ & $0.25-0.5$ & $0.06-2.00$ \\
\hline & & Linezolid $48,50,54$ & $\mathrm{I}-2$ & $1-4$ & 0.5 to $>8.0$ \\
\hline & & Vancomycin ${ }^{50,54}$ & $>32-128$ & $>32$ to $>128$ & 8 to $>128$ \\
\hline & & Cefotaxime $^{48}$ & $>64$ & $>64$ & $>64$ \\
\hline & & Levofloxacin ${ }^{48,54}$ & $>16-64$ & $>16-128$ & $\mathrm{I}-128$ \\
\hline & & Ampicillin ${ }^{54}$ & $>128$ & $>128$ & 64 to $>128$ \\
\hline & & Erythromycin ${ }^{54}$ & 128 & $>128$ & 64 to $>128$ \\
\hline & & Tetracycline ${ }^{54}$ & 0.25 & 128 & $\leq 0.06-128.00$ \\
\hline & & Teicoplanin ${ }^{54}$ & 16 & 64 & $2-64$ \\
\hline \multirow[t]{3}{*}{ E. faecium $R$ to linezolid } & $36^{51}+45^{53}$ & TR-700 51,53 & 2 & $2-4$ & $0.5-8.0$ \\
\hline & & Linezolid $^{51}$ & 32 & 64 & 4 to $>128$ \\
\hline & & Vancomycin ${ }^{53}$ & $>16$ & $>16$ & 0.5 to $>16$ \\
\hline \multirow[t]{4}{*}{ Listeria monocytogenes } & $33^{48}$ & Tedizolid & 0.25 & 0.25 & $0.25-0.50$ \\
\hline & & Cefotaxime & 32 & 32 & $2-32$ \\
\hline & & Levofloxacin & 1 & 1 & $\mathrm{I}-2$ \\
\hline & & Linezolid & 2 & 2 & $2-2$ \\
\hline Staphylococcus aureus S & $105^{48}+95^{50}+30^{54}$ & TR-700 $48,50,54$ & $0.25-0.50$ & 0.5 & $0.25-8.00$ \\
\hline \multirow[t]{3}{*}{ to methicillin } & & Linezolid $48,50,54$ & $2-4$ & $2-4$ & I to $>8$ \\
\hline & & Vancomycin ${ }^{48,50,54}$ & $0.5-1.0$ & 1 & $0.25-2.00$ \\
\hline & & Oxacillin ${ }^{48,50,54}$ & $0.25-0.50$ & 0.5 & $0.06-0.50$ \\
\hline
\end{tabular}


Table 4 (Continued)

\begin{tabular}{|c|c|c|c|c|c|}
\hline Microorganism & Strains tested (n) & Antibiotic & $\mathrm{MIC}_{50}(\mathrm{mcg} / \mathrm{mL})$ & $\mathrm{MIC}_{90}(\mathrm{mcg} / \mathrm{mL})$ & Range (mcg/mL) \\
\hline & & Cefotaxime $^{48}$ & 2 & 2 & $0.03-4.00$ \\
\hline & & Levofloxacin ${ }^{48,54}$ & $0.25-0.50$ & $1-4$ & 0.12 to $>16.00$ \\
\hline & & Erythromycin ${ }^{48,54}$ & 0.5 & $>128$ & 0.5 to $>128.0$ \\
\hline & & Clindamycin ${ }^{48,54}$ & 0.25 & 0.25 & $\leq 0.06-1.00$ \\
\hline & & Cotrimoxazole ${ }^{48,54}$ & 0.25 & 2 & $\leq 0.06-32.00$ \\
\hline & & Gentamycin ${ }^{48,54}$ & 0.5 & 128 & 0.06 to $>128.00$ \\
\hline & & Tetracycline $e^{48,54}$ & 0.5 & 32 & $0.25-64.00$ \\
\hline \multirow[t]{2}{*}{ S. aureus $S$ to methicillin** } & $39^{55}$ & Tedizolid & 0.25 & 0.25 & $0.12-0.50$ \\
\hline & & Linezolid & I & 2 & $0.5-2.0$ \\
\hline \multirow[t]{11}{*}{ S. aureus $\mathrm{R}$ to methicillin } & $129^{48}+103^{50}+30^{54}$ & TR-700 $48,50,54$ & 0.5 & $0.5-1.0$ & $0.12-16.0$ \\
\hline & & Linezolid ${ }^{48,50,54}$ & 2 & 4 & I to $>8$ \\
\hline & & Vancomycin ${ }^{50}$ & 1 & 1 & $0.5-2.0$ \\
\hline & & Oxacillin ${ }^{50,54}$ & 32 to $>128$ & 32 to $>128$ & 4 to $>128$ \\
\hline & & Cefotaxime $^{48}$ & 16 & $>64$ & 2 to $>64$ \\
\hline & & Levofloxacin ${ }^{48,54}$ & $8-16$ & $>16$ to $>128$ & 0.12 to $>128.00$ \\
\hline & & Erythromycin 48,54 & $>128$ & $>128$ & 0.5 to $>128.0$ \\
\hline & & Clindamycin ${ }^{48,54}$ & $>128$ & $>128$ & 0.25 to $>128.00$ \\
\hline & & Cotrimoxazole $e^{48,54}$ & 0.5 & $>128$ & 0.25 to $>128.00$ \\
\hline & & Gentamycin ${ }^{48,54}$ & 64 & $>128$ & 0.25 to $>128.00$ \\
\hline & & Tetracycline $e^{48,54}$ & 64 & 64 & $0.5-128.0$ \\
\hline S. aureus $\mathrm{R}$ to methicillin & $100^{50}$ & TR-700 & 0.5 & 0.5 & $0.25-1.00$ \\
\hline \multirow[t]{3}{*}{ community-acquired } & & Linezolid & 2 & 4 & $\mathrm{I}-4$ \\
\hline & & Vancomycin & 4 & 4 & $\mathrm{I}-4$ \\
\hline & & Oxacillin & 32 & 32 & 4 to $>32$ \\
\hline \multirow[t]{2}{*}{ S. aureus $\mathrm{R}$ to methicillin** } & $124^{55}$ & Tedizolid & 0.25 & 0.25 & $0.12-0.50$ \\
\hline & & Linezolid & I & 2 & $0.5-2.0$ \\
\hline \multirow[t]{5}{*}{ S. aureus $\mathbf{S}$ to linezolid* } & $449^{49}$ & TR-700 & 0.25 & 0.5 & $0.125-0.500$ \\
\hline & & Linezolid & 2 & 2 & $\leq 0.25-4.00$ \\
\hline & & Daptomycin & 0.25 & 0.5 & $\leq 0.125-1.000$ \\
\hline & & Vancomycin & I & I & $\leq 0.5-4.0$ \\
\hline & & Teicoplanin & $\leq 0.5$ & I & $\leq 0.5-4.0$ \\
\hline$-S$. aureus $S$ to linezolid, & $202^{49}$ & TR-700 & 0.25 & 0.25 & $0.125-0.500$ \\
\hline \multirow[t]{4}{*}{ S to oxacillin* } & & Linezolid & I & 2 & $\leq 0.25-4.00$ \\
\hline & & Daptomycin & 0.25 & 0.5 & $\leq 0.125-1.000$ \\
\hline & & Vancomycin & 1 & 1 & $\leq 0.5-2.0$ \\
\hline & & Teicoplanin & $\leq 0.5$ & 1 & $\leq 0.5-2.0$ \\
\hline$-S$. aureus $S$ to linezolid, & $247^{49}$ & TR-700 & 0.25 & 0.5 & $0.125-0.500$ \\
\hline \multirow[t]{4}{*}{ R to oxacillin* } & & Linezolid & 2 & 4 & $\leq 0.25-4.00$ \\
\hline & & Daptomycin & 0.5 & 0.5 & $\leq 0.125-1.000$ \\
\hline & & Vancomycin & 1 & 2 & $\leq 0.5-4.0$ \\
\hline & & Teicoplanin & $\leq 0.5$ & 1 & $\leq 0.5-4.0$ \\
\hline \multirow[t]{5}{*}{ S. aureus R to linezolid* } & $7^{49}$ & TR-700 & 0.5 & NA & 0.5 \\
\hline & & Linezolid & 16 & NA & 16 \\
\hline & & Daptomycin & 0.5 & NA & 0.5 \\
\hline & & Vancomycin & 2 & NA & $\mathrm{I}-2$ \\
\hline & & Teicoplanin & I & NA & $\leq 0.5-2.0$ \\
\hline \multirow[t]{9}{*}{ S. aureus R to linezolid } & $13^{48}+17^{51}+5^{52}$ & Tedizolid $^{48,51,52}$ & $0.25-4.00$ & $0.2-8.0, N^{52}$ & $0.12-16.00$ \\
\hline & & Cefotaxime $^{48}$ & $>64$ & $>64$ & 2 to $>64$ \\
\hline & & Levofloxacin $^{48}$ & $>16$ & $>16$ & 0.25 to $>16.00$ \\
\hline & & Linezolid ${ }^{48,51,52}$ & 2 to $>8$ & $>8-16, N^{52}$ & $\mathrm{I}-64$ \\
\hline & & Vancomycin ${ }^{52}$ & I & NA & I \\
\hline & & Teicoplanin ${ }^{52}$ & I & NA & $\leq 0.5-2.0$ \\
\hline & & Daptomycin ${ }^{52}$ & 0.5 & NA & 0.5 \\
\hline & & Tigecycline $^{52}$ & 0.12 & NA & $0.12-0.25$ \\
\hline & & $\begin{array}{l}\text { Quinupristin/ } \\
\text { dalfopristin }\end{array}$ & 4 & NA & $\mathrm{I}-4$ \\
\hline
\end{tabular}


Table 4 (Continued)

\begin{tabular}{|c|c|c|c|c|c|}
\hline Microorganism & Strains tested $(n)$ & Antibiotic & $\mathrm{MIC}_{50}(\mathrm{mcg} / \mathrm{mL})$ & $\mathrm{MIC}_{90}(\mathrm{mcg} / \mathrm{mL})$ & Range $(\mathrm{mcg} / \mathrm{mL})$ \\
\hline & & Ciprofloxacin ${ }^{52}$ & $>4$ & NA & $\leq \mathrm{I}$ to $>4$ \\
\hline & & Cotrimoxazole ${ }^{52}$ & $\leq 1$ & NA & $\leq 1$ \\
\hline & & Tetracycline ${ }^{52}$ & $\leq 4$ & NA & $\leq 4$ \\
\hline & & Erythromycin ${ }^{52}$ & $>4$ & NA & $\leq 0.5$ to $>4.0$ \\
\hline & & Clindamycin ${ }^{52}$ & $>4$ & NA & $>4$ \\
\hline & & Chloramphenicol ${ }^{52}$ & $>32$ & NA & 16 to $>32$ \\
\hline & & Gentamycin $^{52}$ & $\leq 4$ & NA & $\leq 4$ to $>16$ \\
\hline & & Tobramycin ${ }^{52}$ & $>16$ & NA & $\leq 4$ to $>16$ \\
\hline & & Rifampicin $^{52}$ & $\leq 1$ & NA & $\leq \mathrm{I}$ to $>4$ \\
\hline \multirow[t]{4}{*}{ S. aureus not $S$ to vancomycin } & $32^{48}$ & Tedizolid & 0.25 & 1 & $0.12-1.00$ \\
\hline & & Cefotaxime & $>64$ & $>64$ & 2 to $>64$ \\
\hline & & Levofloxacin & 16 & $>16$ & 4 to $>16$ \\
\hline & & Linezolid & 2 & 4 & $\mathrm{I}-4$ \\
\hline \multirow[t]{2}{*}{ Coagulase-negative staphylococci** } & $7^{55}$ & Tedizolid & NA & NA & $0.12-0.25$ \\
\hline & & Linezolid & NA & NA & $0.5-1.0$ \\
\hline Coagulase-negative & $199^{\mathrm{a}, 49}$ & TR-700 & 0.25 & 0.25 & $\leq 0.03-0.50$ \\
\hline \multirow[t]{4}{*}{ staphylococci S to linezolid* } & & Linezolid & I & 2 & $\leq 0.25-4.00$ \\
\hline & & Daptomycin & 0.25 & 0.5 & $\leq 0.125-1.000$ \\
\hline & & Vancomycin & 2 & 2 & $\leq 0.5-4.0$ \\
\hline & & Teicoplanin & 2 & 8 & $\leq 0.5-32.0$ \\
\hline -Coagulase-negative staphylococci & $41^{49}$ & TR-700 & 0.25 & 0.25 & $0.06-0.25$ \\
\hline \multirow[t]{4}{*}{ S to linezolid, $S$ to oxacillin* } & & Linezolid & I & 2 & $\leq 0.25-2.00$ \\
\hline & & Daptomycin & 0.25 & 0.5 & $\leq 0.125-0.500$ \\
\hline & & Vancomycin & 2 & 2 & $\leq 0.5-2.0$ \\
\hline & & Teicoplanin & 2 & 4 & $\leq 0.5-8.0$ \\
\hline -Coagulase-negative staphylococci S & $158^{49}$ & TR-700 & 0.125 & 0.25 & $\leq 0.03-0.50$ \\
\hline \multirow[t]{4}{*}{ to linezolid, R to oxacillin* } & & Linezolid & 1 & 2 & $\leq 0.25-4.00$ \\
\hline & & Daptomycin & 0.25 & 0.5 & $\leq 0.125-1.000$ \\
\hline & & Vancomycin & 2 & 2 & $\leq 0.5-4.0$ \\
\hline & & Teicoplanin & 2 & 8 & $\leq 0.5-32.0$ \\
\hline Coagulase-negative staphylococci & $5^{\mathrm{b}, 49}$ & TR-700 & 2 & NA & $0.25-4.00$ \\
\hline \multirow[t]{4}{*}{$\mathrm{R}$ to linezolid } & & Linezolid & 16 & NA & $16-256$ \\
\hline & & Daptomycin & 0.5 & NA & $0.25-0.50$ \\
\hline & & Vancomycin & 2 & NA & $\mathrm{I}-2$ \\
\hline & & Teicoplanin & 4 & NA & $1-16$ \\
\hline Coagulase-negative staphylococci & $46^{48}+29^{54}$ & Tedizolid ${ }^{48,54}$ & $0.25-0.50$ & 0.5 & $0.12-1.0$ \\
\hline \multirow[t]{10}{*}{$S$ to methicillin } & & Cefotaxime $^{48}$ & 0.5 & 2 & $0.03-4.00$ \\
\hline & & Levofloxacin ${ }^{48,54}$ & $0.25-0.50$ & 0.5 & $0.06-32.00$ \\
\hline & & Linezolid $^{48,54}$ & $\mathrm{I}-2$ & $2-4$ & $0.5-4.0$ \\
\hline & & Erythromycin ${ }^{54}$ & 0.5 & 128 & 0.25 to $>128.00$ \\
\hline & & Clindamycin ${ }^{54}$ & 0.25 & 1 & 0.12 to $>128.00$ \\
\hline & & Cotrimoxazole ${ }^{54}$ & 0.25 & 16 & $\leq 0.06-32.00$ \\
\hline & & Gentamycin $^{54}$ & 0.12 & 64 & $0.06-128.00$ \\
\hline & & Tetracycline ${ }^{54}$ & 0.5 & 32 & $0.5-128.0$ \\
\hline & & Oxacillin ${ }^{54}$ & 0.12 & 0.25 & $0.06-0.25$ \\
\hline & & Vancomycin ${ }^{54}$ & 1 & 1 & $0.5-2.0$ \\
\hline Coagulase-negative staphylococci & $58^{48}+26^{54}$ & Tedizolid ${ }^{48,54}$ & $0.25-0.50$ & 0.5 & $0.12-1.00$ \\
\hline \multirow[t]{7}{*}{$\mathrm{R}$ to methicillin } & & Cefotaxime $^{48}$ & 8 & $>64$ & 0.5 to $>64.0$ \\
\hline & & Levofloxacin ${ }^{48,54}$ & $0.5-8.0$ & $\geq 16$ & 0.12 to $>16.00$ \\
\hline & & Linezolid $^{48,54}$ & $\mathrm{I}-2$ & $2-4$ & $0.5-8.0$ \\
\hline & & Erythromycin ${ }^{54}$ & 64 & 128 & $\leq 0.06$ to $>128.00$ \\
\hline & & Clindamycin ${ }^{54}$ & 0.25 & $>128$ & 0.12 to $>128.00$ \\
\hline & & Cotrimoxazole ${ }^{54}$ & 2 & 32 & $\leq 0.06-32.00$ \\
\hline & & Gentamycin $^{54}$ & 16 & 64 & $0.06-128.00$ \\
\hline
\end{tabular}


Table 4 (Continued)

\begin{tabular}{|c|c|c|c|c|c|}
\hline Microorganism & Strains tested $(n)$ & Antibiotic & $\mathrm{MIC}_{50}(\mathrm{mcg} / \mathrm{mL})$ & $\mathrm{MIC}_{90}(\mathrm{mcg} / \mathrm{mL})$ & Range (mcg/mL) \\
\hline & & Tetracycline ${ }^{54}$ & 4 & 128 & 0.5 to $>128.0$ \\
\hline & & Oxacillin ${ }^{54}$ & 4 & 64 & 0.5 to $>128.0$ \\
\hline & & Vancomycin ${ }^{54}$ & 1 & 2 & $0.25-2.00$ \\
\hline Coagulase-negative staphylococci & $40^{c, 53}$ & TR-700 & 4 & 16 & I to $>32$ \\
\hline not $S$ to linezolid & & Vancomycin & 2 & 2 & $\mathrm{I}-2$ \\
\hline Coagulase-negative staphylococci & $6^{\mathrm{d}, 51}+164^{\mathrm{e}, 52}$ & TR-700 51,52 & $N A,{ }^{51} 4^{52}$ & $N A,{ }^{51} 8^{52}$ & $0.06-16.00$ \\
\hline \multirow[t]{15}{*}{$R$ to linezolid } & & Linezolid ${ }^{51,52}$ & $N A,{ }^{51} 128^{52}$ & $N A,{ }^{51}>128^{52}$ & 8 to $>128$ \\
\hline & & Vancomycin ${ }^{52}$ & 2 & 4 & $1-4$ \\
\hline & & Teicoplanin ${ }^{52}$ & 4 & 16 & $\leq 0.5-64$ \\
\hline & & Daptomycin ${ }^{52}$ & 0.5 & 1 & $0.25-2.00$ \\
\hline & & Tigecycline ${ }^{52}$ & 0.25 & 0.5 & $\leq 0.06-0.50$ \\
\hline & & $\begin{array}{l}\text { Quinupristin/ } \\
\text { dalfopristin }^{52}\end{array}$ & I & 2 & $\leq 0.25-16.00$ \\
\hline & & Ciprofloxacin ${ }^{52}$ & $>4$ & $>4$ & $\leq \mathrm{I}$ to $>4$ \\
\hline & & Cotrimoxazole ${ }^{52}$ & $>4$ & $>4$ & $\leq \mathrm{I}$ to $>4$ \\
\hline & & Tetracycline ${ }^{52}$ & $\leq 4$ & $\leq 4$ & $\leq 4$ to $>16$ \\
\hline & & Erythromycin ${ }^{52}$ & $>4$ & $>4$ & $\leq 0.5$ to $>4.0$ \\
\hline & & Clindamycin ${ }^{52}$ & $>4$ & $>4$ & $\leq 0.5$ to $>4.0$ \\
\hline & & Chloramphenicol ${ }^{52}$ & $>32$ & $>32$ & $\leq 8$ to $>32$ \\
\hline & & Gentamycin $^{52}$ & $>16$ & $>16$ & $\leq 4$ to $>16$ \\
\hline & & Tobramycin ${ }^{52}$ & $>16$ & $>16$ & $\leq 4$ to $>16$ \\
\hline & & Rifampicin $^{52}$ & $>4$ & $>4$ & $\leq \mathrm{I}$ to $>4$ \\
\hline Staphylococcus epidermidis & $48^{50}$ & TR-700 & 0.25 & 0.5 & $0.12-1.00$ \\
\hline \multirow[t]{3}{*}{$S$ to methicillin } & & Linezolid & I & 2 & $0.5-4.0$ \\
\hline & & Vancomycin & 2 & 2 & $\mathrm{I}-4$ \\
\hline & & Oxacillin & 0.12 & 0.25 & $0.06-0.25$ \\
\hline \multirow[t]{4}{*}{ S. epidermidis $\mathrm{R}$ to methicillin } & $72^{50}$ & TR-700 & 0.25 & 0.5 & $0.12-1.00$ \\
\hline & & Linezolid & 1 & 2 & $0.5-4.0$ \\
\hline & & Vancomycin & 2 & 2 & $0.25-4$ \\
\hline & & Oxacillin & 16 & $>32$ & $0.5 \geq 32$ \\
\hline \multirow[t]{2}{*}{ S. epidermidis R to linezolid } & $19^{51}$ & TR-700 & 4 & 8 & 2 to $>64$ \\
\hline & & Linezolid & 32 & $>128$ & 16 to $>128$ \\
\hline \multirow[t]{4}{*}{ Beta-hemolytic streptococci } & $202^{f, 48}$ & Tedizolid & 0.25 & 0.25 & $0.12-0.5$ \\
\hline & & Cefotaxime & 0.03 & 0.06 & $0.015-0.06$ \\
\hline & & Levofloxacin & 0.5 & I & $0.25-2.00$ \\
\hline & & Linezolid & 1 & 2 & $\mathrm{I}-4$ \\
\hline \multirow[t]{8}{*}{ Streptococcus agalactiae } & $52^{50}+15^{54}$ & TR-700 00,54 & 0.25 & 0.5 & $0.06-1.00$ \\
\hline & & Linezolid $^{50,54}$ & 2 & 2 & $\mathrm{I}-2$ \\
\hline & & Vancomycin ${ }^{50}$ & 0.5 & 0.5 & $0.25-1.00$ \\
\hline & & Penicillin $\mathrm{G}^{54}$ & 0.06 & 0.06 & $0.03-0.06$ \\
\hline & & Cefotaxime $^{54}$ & 0.06 & 0.06 & $0.03-0.06$ \\
\hline & & Clindamycin $^{54}$ & 0.25 & $>128$ & 0.25 to $>128.00$ \\
\hline & & Erythromycin ${ }^{54}$ & 0.5 & $>128$ & 0.25 to $>128.00$ \\
\hline & & Levofloxacin ${ }^{54}$ & 1 & 2 & $\mathrm{I}-2$ \\
\hline Streptococcus pneumoniae & $53^{48}+38^{50}$ & TR-700 48,50 & 0.25 & 0.25 & $0.03-0.50$ \\
\hline \multirow[t]{4}{*}{ S to penicillin } & & Linezolid $^{48,50}$ & I & $\mathrm{I}-2$ & $0.12-2.00$ \\
\hline & & Vancomycin ${ }^{50}$ & 0.25 & 0.5 & $0.12-1.00$ \\
\hline & & Cefotaxime $^{48}$ & 0.015 & 0.03 & $0.015-0.250$ \\
\hline & & Levofloxacin ${ }^{48}$ & I & 1 & $0.25-4.00$ \\
\hline \multirow[t]{5}{*}{ S. pneumoniae I to penicillin } & $26^{48}+37^{50}$ & TR-700 48,50 & 0.25 & $0.25-0.50$ & $0.06-0.50$ \\
\hline & & Linezolid $^{48,50}$ & I & $\mathrm{I}-2$ & $0.5-4.0$ \\
\hline & & Vancomycin ${ }^{50}$ & 0.25 & 0.5 & $0.25-0.50$ \\
\hline & & Cefotaxime $^{48}$ & 0.12 & 0.5 & $0.03-1.00$ \\
\hline & & Levofloxacin ${ }^{48}$ & I & I & $0.5-1.0$ \\
\hline
\end{tabular}


Table 4 (Continued)

\begin{tabular}{|c|c|c|c|c|c|}
\hline Microorganism & Strains tested (n) & Antibiotic & $\mathrm{MIC}_{50}(\mathrm{mcg} / \mathrm{mL})$ & $\mathrm{MIC}_{90}(\mathrm{mcg} / \mathrm{mL})$ & Range (mcg/mL) \\
\hline \multirow[t]{5}{*}{ S. pneumoniae $\mathrm{R}$ to penicillin } & $54^{48}+35^{50}$ & TR-700 48,50 & 0.25 & 0.25 & $0.06-0.50$ \\
\hline & & Linezolid ${ }^{48,50}$ & I & $1-2$ & $0.25-2.00$ \\
\hline & & Vancomycin $^{50}$ & 0.25 & 0.5 & $0.12-1.00$ \\
\hline & & Cefotaxime $^{48}$ & I & 8 & $0.5-8.0$ \\
\hline & & Levofloxacin $^{48}$ & I & 1 & $0.5-2.0$ \\
\hline \multirow[t]{8}{*}{ Streptococcus pyogenes } & $102^{50}+15^{54}$ & TR-700 50,54 & $0.12-0.25$ & $0.25-0.50$ & $0.06-0.50$ \\
\hline & & Linezolid $^{50,54}$ & I & 2 & $0.06-2.00$ \\
\hline & & Vancomycin ${ }^{50}$ & 0.5 & 0.5 & $0.25-1.00$ \\
\hline & & Penicillin $\mathrm{G}^{54}$ & 0.015 & 0.015 & $\leq 0.008-0.015$ \\
\hline & & Cefotaxime $^{54}$ & 0.015 & 0.03 & $\leq 0.008-0.030$ \\
\hline & & Clindamycin ${ }^{54}$ & 0.12 & 0.25 & $0.12-0.25$ \\
\hline & & Erythromycin ${ }^{54}$ & 0.12 & 0.25 & $0.12-0.25$ \\
\hline & & Levofloxacin ${ }^{54}$ & I & 4 & $0.5-4$ \\
\hline \multirow[t]{4}{*}{ Streptococcus viridans } & $30^{48}$ & Tedizolid & 0.25 & 0.25 & $0.06-0.50$ \\
\hline & & Cefotaxime & 0.12 & 0.5 & $0.015-2.000$ \\
\hline & & Levofloxacin & I & 2 & $0.25-2.00$ \\
\hline & & Linezolid & 2 & 2 & $0.5-2.0$ \\
\hline
\end{tabular}

Notes: *Blood culture strains; **skin and skin-structure infection strains; aStaphylococcus epidermidis: 135, Staphylococcus hominis: 40, Staphylococcus haemolyticus: 19, Staphylococcus lugdunensis: 3, Staphylococcus intermedius: I, Staphylococcus warneri: I; 'b. epidermidis: 2, S. hominis: 2, S. haemolyticus: I; 'Staphylococcus capitis: 2, S. epidermidis: 29, S. haemolyticus: 5, Staphylococcus simulans: 2, Staphylococcus xylosus: 2; ${ }^{d}$ S. hominis: I, Staphylococcus sciuri: 5; e S. epidermidis: I42, S. hominis: I5, S. warneri: 7; fStreptococcus agalactiae: 10I, Streptococcus pyogenes: I0I. In bold: Staphylococcus aureus S to linezolid* group includes 202 strains of S. aureus S to linezolid, S to oxacillin* and 247 strains of S. aureus S to linezolid, R to oxacillin*. Coagulase-negative staphylococci S to linezolid* group includes 4 I strains of coagulase-negative staphylococci $S$ to linezolid, S to oxacillin* and 158 strains of coagulase-negative staphylococci $S$ to linezolid, R to oxacillin*. Data from Brown and Traczewski, ${ }^{48}$ Betriu et al, ${ }^{49}$ Schaadt et al, ${ }^{50}$ Shaw et al, ${ }^{51}$ Rodríguez-Avial et al, ${ }^{52}$ Jones et al, ${ }^{53}$ Yum et al, ${ }^{54}$ Prokocimer et al. ${ }^{55}$

Abbreviations: $\mathrm{S}$, sensitive; I, intermediate sensitivity; R, resistant; NA, not applicable; $\mathrm{MIC}_{5}$, minimum inhibitory concentration required to inhibit the growth of $50 \%$ of organisms; $\mathrm{MIC}_{90}$, minimum inhibitory concentration required to inhibit the growth of $90 \%$ of organisms.

between 2 and $4 \mathrm{mcg} / \mathrm{mL}$ against seven strains of Enterococcus faecium and one strain of Enterococcus faecalis carrying the G2576U mutation, compared with an MIC for linezolid of between 16 and $32 \mathrm{mcg} / \mathrm{mL} .{ }^{51}$ Another study reported MIC values for tedizolid between 0.5 and $8.0 \mathrm{mcg} / \mathrm{mL}$ when tested against 69 strains of Enterococcus spp. carrying the G2576T mutation (MIC of linezolid 4 to $>32 \mathrm{mcg} / \mathrm{mL}$ ). ${ }^{53}$

In another study, resistance to tedizolid was observed in only three of the 36 strains of Enterococcus spp. resistant to linezolid analyzed. ${ }^{57}$ Two of them were strains of $E$. faecium homozygous for the G2576T mutation (MIC against tedizolid of $8 \mathrm{mcg} / \mathrm{mL}$ ), while no resistance mechanism could be established in the third strain of this same species (MIC against tedizolid of $16 \mathrm{mcg} / \mathrm{mL}$ ).

\section{Aerobic Gram-negative microorganisms}

Tedizolid shows a limited activity against strains of Haemophilus influenzae and Moraxella catarrhalis $\left(\mathrm{MIC}_{90}\right.$ of 16 and $4 \mathrm{mcg} / \mathrm{mL}$, respectively) (Table 6). ${ }^{50}$ Both tedizolid and linezolid have shown a lower activity than that of cefotaxime and levofloxacin against strains of these microorganisms. ${ }^{48}$ However, the $\mathrm{MIC}_{90}$ of tedizolid was two times lower than that observed for linezolid $\left(\mathrm{MIC}_{90}\right.$ for linezolid of 32 and $8 \mathrm{mcg} / \mathrm{mL}$, respectively). ${ }^{50}$

\section{Anaerobic microorganisms}

The activity of tedizolid against anaerobic microorganisms has been reported to be greater than or equal to that observed with linezolid (Table 7). ${ }^{50}$

\section{Acid-fast bacilli}

Tedizolid has shown excellent activity against 95 strains of Mycobacterium tuberculosis, nine of which showed resistance to isoniazid or rifampicin and 25 to both tuberculostatics. ${ }^{58}$ The $\mathrm{MIC}_{50}$ was $0.25 \mathrm{mcg} / \mathrm{mL}$ and the $\mathrm{MIC}_{90}$ was $0.50 \mathrm{mcg} / \mathrm{mL}$ for all the strains. Additionally, the $\mathrm{MIC}_{50}$ and $\mathrm{MIC}_{90}$ values of tedizolid phosphate were $0.5 \mathrm{mcg} / \mathrm{mL}$ against ten strains of sensitive $M$. tuberculosis and ten strains of $M$. tuberculosis resistant to tuberculostatics.

\section{Resistance mechanisms}

Several oxazolidinone resistance mechanisms have been described, including mutations in domain $\mathrm{V}$ of $23 \mathrm{~S}$ rRNA ${ }^{59,60}$ and horizontal transmission of the $c f r$ gene. ${ }^{17,18}$ However, a number of ribosomal proteins of the 50S subunit have regions that interact with the oxazolidinone-binding site in the peptidyl transferase center, most notably L3 and L4. Mutations in the genes that encode these proteins may have an impact on the sensitivity presented by different microorganisms to this family 
Table 5 Activity of tedizolid (TR-700) and other antibiotics against Gram-positive microorganisms with characterized linezolidresistance mechanisms

\begin{tabular}{|c|c|c|c|c|c|}
\hline Microorganism & Strains tested (n) & Antibiotic & $\mathrm{MIC}_{50}(\mathrm{mcg} / \mathrm{mL})$ & $\mathrm{MIC}_{90}(\mathrm{mcg} / \mathrm{mL})$ & Range (mcg/mL) \\
\hline \multicolumn{6}{|l|}{ G2576T } \\
\hline \multirow[t]{9}{*}{ Enterococcus spp. } & $69^{a, 53}$ & TR-700 & 2 & 2 & $0.5-8$ \\
\hline & & Linezolid & 8 & 16 & $4-32$ \\
\hline & & Ciprofloxacin & $>4$ & $>4$ & I to $>4$ \\
\hline & & Daptomycin & I & 2 & $0.12-4.00$ \\
\hline & & Erythromycin & $>2$ & $>2$ & $\leq 0.25$ to $>2.0$ \\
\hline & & Quinupristin/dalfopristin & $\mathrm{I}$ & $>2$ & $\leq 0.25$ to $>2.0$ \\
\hline & & Teicoplanin & $>16$ & $>16$ & $\leq 0.12$ to $>16.00$ \\
\hline & & Tetracycline & $\leq 2$ & $>8$ & $\leq 2$ to $>8$ \\
\hline & & Vancomycin & $>16$ & $>16$ & 0.5 to $>16.0$ \\
\hline \multirow[t]{13}{*}{ Staphylococcus spp. } & $39^{\mathrm{b}, 53}$ & TR-700 & 4 & 8 & $0.5-16.0$ \\
\hline & & Linezolid & 16 & $>32$ & $8 \geq 32$ \\
\hline & & Ciprofloxacin & $>4$ & $>4$ & 0.25 to $>4.00$ \\
\hline & & Clindamycin & I & $>2$ & $\leq 0.25$ to $>2.00$ \\
\hline & & Daptomycin & 0.25 & 0.5 & $0.25-1.00$ \\
\hline & & Erythromycin & $>2$ & $>2$ & $\leq 0.25$ to $>2.00$ \\
\hline & & Gentamicin & $>8$ & $>8$ & $\leq 2$ to $>8$ \\
\hline & & Oxacillin & $>2$ & $>2$ & $\leq 0.25$ to $>2.00$ \\
\hline & & Quinupristin/dalfopristin & 0.5 & 1 & $\leq 0.25-2.00$ \\
\hline & & Teicoplanin & 4 & 8 & $0.5-16.0$ \\
\hline & & Tetracycline & $\leq 2$ & $>8$ & $\leq 2$ to $>8$ \\
\hline & & Trimethoprim/sulfamethoxazole & $>2$ & $>2$ & $\leq 0.5$ to $>2.0$ \\
\hline & & Vancomycin & 2 & 2 & $0.5-2.0$ \\
\hline \multicolumn{6}{|l|}{ crf gene } \\
\hline \multirow[t]{13}{*}{ Staphylococcus spp. } & $4^{c, 53}$ & TR-700 & 1 & - & $0.5-8.0$ \\
\hline & & Linezolid & 32 & - & 8 to $>32$ \\
\hline & & Ciprofloxacin & $>4$ & - & $>4$ \\
\hline & & Clindamycin & $>4$ & - & $>4$ \\
\hline & & Daptomycin & $\leq 0.25$ & - & $\leq 0.25$ \\
\hline & & Erythromycin & $\leq 0.25$ & - & $\leq 0.25$ to $>2.00$ \\
\hline & & Gentamicin & $>8$ & - & $\leq 2$ to $>8$ \\
\hline & & Oxacillin & $>2$ & - & $>2$ \\
\hline & & Quinupristin/dalfopristin & $>2$ & - & I to $>2$ \\
\hline & & Teicoplanin & $\leq 2$ & - & $\leq 2$ \\
\hline & & Tetracycline & $\leq 2$ & - & $\leq 2$ to $>8$ \\
\hline & & Trimethoprim/sulfamethoxazole & $>2$ & - & $\leq 0.5$ to $>2.0$ \\
\hline & & Vancomycin & I & - & $\mathrm{I}-2$ \\
\hline
\end{tabular}

Notes: aEnterococcus faecalis: 15, Enterococcus faecium: 54; ' Staphylococcus aureus: 6, Staphylococcus epidermidis: 22, Staphylococcus capitis: 2, Staphylococcus haemolyticus: 5, Staphylococcus simulans: 2, Staphylococcus xylosus: 2; ' 5 . aureus: 2, S. epidermidis: 2.

Data from Jones et al..$^{53}$

Abbreviations: $\mathrm{MIC}_{50}$, minimum inhibitory concentration required to inhibit the growth of $50 \%$ of organisms; $\mathrm{MIC}_{90}$, minimum inhibitory concentration required to inhibit the growth of $90 \%$ of organisms.

of antibiotics. L3 mutations were first associated with reduced susceptibility to oxazolidinones in a study that sequenced the $r p l C$ gene, encoding L3, in eleven clinical isolates resistant to linezolid ${ }^{61}$ The sequence analysis identified two L3 mutations, $\Delta$ Ser145 in S. aureus strain NRS127 and Ala157Arg in Staphylococcus epidermidis strain 1653059 , both adjacent to the oxazolidinone-binding site in the peptidyl transferase center. Tedizolid maintained an eight- to 16-fold potency advantage over linezolid against strains NRS127 (MIC $1 \mathrm{vs} 8 \mathrm{mcg}$ / $\mathrm{mL}$ ) and 1653059 (MIC $16 \mathrm{vs} 256 \mathrm{mcg} / \mathrm{mL}$ ). Another study of MRSA clinical isolates resistant to linezolid and carrying the $c f r$ gene identified mutations in L3, including $\Delta$ Ser145/ His146Tyr and $\Delta$ Met169-Gly174. ${ }^{62}$ The MICs of tedizolid were between 1 and $2 \mathrm{mcg} / \mathrm{mL}$, while those of linezolid were between 32 and $64 \mathrm{mcg} / \mathrm{mL}$, respectively.

A study analyzed the structure-activity relationship of different oxazolidinones against strains of $S$. aureus resistant to linezolid due to ribosomal mutations (23S rRNA, L3, L4) or due to methylation of 23S rRNA via horizontal transfer of the $c f r$ gene.$^{63}$ According to the MIC values observed in this 
Table 6 Activity of tedizolid and other antibiotics against Gram-negative microorganisms

\begin{tabular}{|c|c|c|c|c|c|}
\hline Microorganism & Strains tested $(n)$ & Antibiotic & $\mathrm{MIC}_{50}(\mathrm{mcg} / \mathrm{mL})$ & $\mathrm{MIC}_{90}(\mathrm{mcg} / \mathrm{mL})$ & Range $(\mathrm{mcg} / \mathrm{mL})$ \\
\hline \multirow[t]{11}{*}{ All Haemophilus influenzae } & $99^{48}+25^{54}$ & Tedizolid ${ }^{48,54}$ & $2-8$ & $4-16$ & $2-32$ \\
\hline & & Cefotaxime $\mathrm{e}^{48,54}$ & $0.015-0.030$ & 0.5 & $\leq 0.008-2.000$ \\
\hline & & Levofloxacin ${ }^{48,54}$ & 0.12 & 0.12 & 0.12 \\
\hline & & Linezolid $^{48,54}$ & $\geq 8$ & $>8-16$ & $4-16$ \\
\hline & & Ampicillin $^{54}$ & $>128$ & $>128$ & 0.5 to $>128.0$ \\
\hline & & Ampicillin/sulbactam ${ }^{54}$ & 4 & 8 & $0.5-8.0$ \\
\hline & & Cefaclor ${ }^{54}$ & 4 & $>128$ & 2 to $>128$ \\
\hline & & Cefuroxime $^{54}$ & I & $>128$ & 0.25 to $>128.0$ \\
\hline & & Azithromycin ${ }^{54}$ & 4 & 4 & $2-4$ \\
\hline & & Cotrimoxazol $^{54}$ & 4 & 32 & $\leq 0.06-32.00$ \\
\hline & & Tetraciclina $^{54}$ & 0.5 & 8 & $0.25-32.00$ \\
\hline Beta-lactamase-negative & $32^{48}$ & Tedizolid & 8 & 16 & $4-32$ \\
\hline \multirow[t]{3}{*}{ H. influenzae } & & Cefotaxime & 0.008 & 0.015 & $0.008-0.030$ \\
\hline & & Levofloxacin & 0.12 & 0.12 & 0.12 \\
\hline & & Linezolid & $>8$ & $>8$ & 4 to $>8$ \\
\hline Beta-lactamase-positive & $42^{48}$ & Tedizolid & 8 & 32 & $4-32$ \\
\hline \multirow[t]{3}{*}{ H. influenzae } & & Cefotaxime & 0.015 & 0.015 & $0.008-0.030$ \\
\hline & & Levofloxacin & 0.12 & 0.12 & 0.12 \\
\hline & & Linezolid & $>8$ & $>8$ & 8 to $>8$ \\
\hline Beta-lactamase-negative & $25^{48}$ & Tedizolid & 8 & 16 & $2-16$ \\
\hline H. influenzae, not sensitive & & Cefotaxime & 0.5 & 0.5 & $0.03-2.00$ \\
\hline \multirow[t]{2}{*}{ to ampicillin } & & Levofloxacin & 0.12 & 0.12 & 0.12 \\
\hline & & Linezolid & $>8$ & $>8$ & $>8$ to $>8$ \\
\hline \multirow[t]{9}{*}{ Moraxella catarrhalis } & $50^{48}+27^{54}$ & Tedizolid 48,54 & $\mathrm{I}-4$ & $1-4$ & $0.5-4$ \\
\hline & & Cefotaxime $^{48}$ & 0.250 & 1 & $0.03-2$ \\
\hline & & Levofloxacin ${ }^{48,54}$ & 0.06 & 0.06 & $0.03-0.06$ \\
\hline & & Linezolid ${ }^{48,54}$ & $4-8$ & $4-8$ & $2-16$ \\
\hline & & Penicillin $G^{54}$ & 16 & 32 & $0.03-32$ \\
\hline & & Cefaclor ${ }^{54}$ & 2 & 8 & $0.25-32$ \\
\hline & & Clindamycin $^{54}$ & 2 & 4 & $\mathrm{I}-4$ \\
\hline & & Erythromycin ${ }^{54}$ & 0.25 & 0.5 & $0.12-0.5$ \\
\hline & & Tetracycline $e^{54}$ & 0.5 & 0.5 & $0.25-16$ \\
\hline
\end{tabular}

Data from Brown and Traczewski ${ }^{48}$ and Yum et al. ${ }^{54}$

Abbreviations: $\mathrm{MIC}_{50}$, minimum inhibitory concentration required to inhibit the growth of $50 \%$ of organisms; $\mathrm{MIC}_{90}$, minimum inhibitory concentration required to inhibit the growth of $90 \%$ of organisms.

study, the $\mathrm{C}$ and $\mathrm{D}$ rings play a fundamental role in the activity of the antimicrobial, both in strains with ribosomal mutations and in strains that carry the $c f r$ gene. As has been suggested previously, the $\mathrm{C}$ and $\mathrm{D}$ rings of the tedizolid molecule may act as additional hydrogen bond donors to the sugar backbone of residues A2451 and U2584 of rRNA. ${ }^{51}$ Furthermore, the presence of both 1,2,3-triazole and hydroxymethyl substituents on C-5 of ring A maintained the potency of the antibiotic against strains carrying the $c f r$ gene, while the presence of acetamide substituents was associated with a fourfold increase in the MIC value of the antimicrobial. ${ }^{63}$

The structure of linezolid does not contain the $\mathrm{D}$ ring and has an acetamide substituent on C-5 of ring A, unlike tedizolid, whose structure contains a D ring and a hydroxymethyl substituent on C-5 of ring A. These structural features explain why tedizolid maintains activity against strains with high MICs of linezolid, especially those strains that carry the $c f r$ gene. In the same study, the $S$. aureus MIC values of tedizolid were 0.5 to $1.0 \mathrm{mcg} / \mathrm{mL}$ against strains carrying ribosomal mutations (vs 2 to $32 \mathrm{mcg} / \mathrm{mL}$ for linezolid) and $0.5 \mathrm{mcg} / \mathrm{mL}$ against all $c f r$ positive strains tested (vs 2 to $16 \mathrm{mcg} / \mathrm{mL}$ for linezolid). ${ }^{63}$

The potential for $S$. aureus to develop resistance to tedizolid was investigated in a study using representative MSSA and MRSA strains through determination of spontaneous mutation frequencies and by serial passage on antibiotic gradient plates containing tedizolid or linezolid. ${ }^{64}$ The median spontaneous mutation frequency that resulted in a reduction in sensitivity to tedizolid was $1.1 \times 10^{-10}$ for the MSSA strain and $1.9 \times 10^{-10}$ for the MRSA strain. These values were approximately 16-fold lower than those obtained for linezolid $\left(2.0 \times 10^{-9}\right.$ for MSSA and $3.0 \times 10^{-9}$ for MRSA). The spontaneous mutant strains selected with tedizolid possessed the T2500A 23S rRNA mutation or Gly155Arg, Gly155Arg/Met169 Leu, or $\Delta$ Phe127-His146 mutations in 
Table 7 Activity of tedizolid and other antibiotics against anaerobic microorganisms

\begin{tabular}{|c|c|c|c|c|c|}
\hline Microorganism & Strains tested $(n)$ & Antibiotic & $\mathrm{MIC}_{50}(\mathrm{mcg} / \mathrm{mL})$ & $\mathrm{MIC}_{90}(\mathrm{mcg} / \mathrm{mL})$ & Range (mcg/mL) \\
\hline \multirow[t]{11}{*}{ Bacteroides fragilis } & $10^{50}+30^{54}$ & TR-700 50,54 & $2-4$ & $2-4$ & $1-4$ \\
\hline & & Linezolid $^{50,54}$ & 4 & 4 & $2-4$ \\
\hline & & Metronidazole $e^{50,54}$ & $0.12-4.00$ & $1-4$ & 0.12 to $>32.00$ \\
\hline & & Imipenem $^{50,54}$ & $0.12-0.25$ & $0.5-1.0$ & $\leq 0.06-4.00$ \\
\hline & & Ampicillin ${ }^{54}$ & 32 & $>128$ & 16 to $>128$ \\
\hline & & Ampicillin/sulbactam ${ }^{54}$ & 2 & 16 & $\mathrm{I}-32$ \\
\hline & & Piperacillin ${ }^{54}$ & 32 & 256 & 4 to $>256$ \\
\hline & & Piperacillin/tazobactam ${ }^{54}$ & 0.25 & 1 & $0.12-8.00$ \\
\hline & & Cefoxitin $^{54}$ & 8 & 32 & $4-64$ \\
\hline & & Cefotetan ${ }^{54}$ & 8 & 32 & $4-128$ \\
\hline & & Clindamycin ${ }^{54}$ & 128 & $>128$ & $\leq 0.06$ to $>128.00$ \\
\hline \multirow[t]{4}{*}{ Bacteroides vulgatus } & $10^{50}$ & TR-700 & 2 & 4 & $\mathrm{I}-8$ \\
\hline & & Linezolid & 2 & 4 & $2-4$ \\
\hline & & Metronidazole & 0.25 & 0.25 & $0.12-0.50$ \\
\hline & & Imipenem & 0.25 & 0.5 & $0.25-0.50$ \\
\hline Bacteroides & $10^{50}+15^{54}$ & TR-700 50,54 & 2 & 2 & $\mathrm{I}-2$ \\
\hline \multirow[t]{10}{*}{ thetaiotaomicron } & & Linezolid $^{50,54}$ & 4 & $4-8$ & $4-8$ \\
\hline & & Metronidazole $\mathrm{e}^{50,54}$ & $1-4$ & $1-4$ & 0.5 to $>32$ \\
\hline & & Imipenem ${ }^{50,54}$ & $0.25-0.50$ & $1-2$ & $0.12-4$ \\
\hline & & Ampicillin ${ }^{54}$ & 32 & $>128$ & 16 to $>128$ \\
\hline & & Ampicillin/sulbactam ${ }^{54}$ & 1 & 32 & $\mathrm{I}-32$ \\
\hline & & Piperacillin $^{54}$ & 32 & $>256$ & 16 to $>256$ \\
\hline & & Piperacillin/tazobactam ${ }^{54}$ & 4 & 8 & $2-16$ \\
\hline & & Cefoxitin ${ }^{54}$ & 16 & 32 & $16-32$ \\
\hline & & Cefotetan ${ }^{54}$ & 128 & $>128$ & 32 to $>128$ \\
\hline & & Clindamycin ${ }^{54}$ & 8 & $>128$ & 2 to $>128$ \\
\hline \multirow[t]{4}{*}{ Bacteroides ovatus } & $10^{50}$ & TR-700 & 2 & 8 & $0.06-8.00$ \\
\hline & & Linezolid & 8 & 8 & $0.5-8.0$ \\
\hline & & Metronidazole & I & 1 & 0.5 to $>32.0$ \\
\hline & & Imipenem & 0.25 & 0.5 & $0.06-0.50$ \\
\hline \multirow[t]{12}{*}{ Clostridium perfringens } & $10^{50}+15^{54}$ & TR-700 50,54 & $0.25-0.50$ & $0.25-2.0$ & $0.12-2.00$ \\
\hline & & Linezolid ${ }^{50,54}$ & 2 & $2-4$ & $\mathrm{I}-2$ \\
\hline & & Metronidazole $\mathrm{e}^{50,54}$ & $\mathrm{I}-4$ & 4 to $>32$ & I to $>32$ \\
\hline & & Imipenem $^{50,54}$ & $\leq 0.06-0.12$ & $\leq 0.06-0.50$ & $\leq 0.06-1.00$ \\
\hline & & Ampicillin ${ }^{54}$ & $\leq 0.06$ & 0.12 & $\leq 0.06-0.50$ \\
\hline & & Ampicillin/sulbactam ${ }^{54}$ & $\leq 0.06$ & 0.25 & $\leq 0.06-0.50$ \\
\hline & & Piperacillin ${ }^{54}$ & $\leq 0.06$ & 0.25 & $\leq 0.06-1.00$ \\
\hline & & Piperacillin/tazobactam ${ }^{54}$ & $\leq 0.06$ & $\leq 0.06$ & $\leq 0.06$ \\
\hline & & Cefoxitin $^{54}$ & 0.5 & 1 & $0.25-1.00$ \\
\hline & & Cefotetan ${ }^{54}$ & $\leq 0.06$ & 0.12 & $\leq 0.06-0.50$ \\
\hline & & Clindamycin ${ }^{54}$ & $\mathrm{I}$ & 2 & $\leq 0.06-2.00$ \\
\hline & & Vancomycin ${ }^{54}$ & 0.5 & 0.5 & $0.5-2.0$ \\
\hline \multirow[t]{12}{*}{ Peptostreptococcus spp. } & $59^{a, 54}$ & Tedizolid & 0.06 & 0.25 & $0.03-0.25$ \\
\hline & & Linezolid & 0.5 & I & $0.25-2.00$ \\
\hline & & Ampicillin & 0.12 & 1 & $\leq 0.06-16.00$ \\
\hline & & Ampicillin/sulbactam & 0.12 & 1 & $\leq 0.06-8.00$ \\
\hline & & Piperacillin & $\leq 0.06$ & 8 & $\leq 0.06-16.00$ \\
\hline & & Piperacillin/tazobactam & $\leq 0.06$ & 8 & $\leq 0.06-16.00$ \\
\hline & & Cefoxitin & 0.25 & 4 & $\leq 0.06-16.00$ \\
\hline & & Cefotetan & 0.5 & 16 & $\leq 0.06-128.00$ \\
\hline & & Imipenem & $\leq 0.06$ & 0.12 & $\leq 0.06-1.00$ \\
\hline & & Clindamycin & 0.5 & 64 & $\leq 0.06$ to $>128.00$ \\
\hline & & Metronidazole & 1 & 2 & $\leq 0.06-4.00$ \\
\hline & & Vancomycin & 0.25 & 0.5 & $\leq 0.12-1.00$ \\
\hline Peptostreptococcus & $10^{50}$ & TR-700 & 0.25 & 0.5 & $0.12-0.50$ \\
\hline anaerobius & & Linezolid & 1 & 2 & $0.5-8$ \\
\hline
\end{tabular}


Table 7 (Continued)

\begin{tabular}{|c|c|c|c|c|c|}
\hline Microorganism & Strains tested (n) & Antibiotic & $\mathrm{MIC}_{50}(\mathrm{mcg} / \mathrm{mL})$ & $\mathrm{MIC}_{90}(\mathrm{mcg} / \mathrm{mL})$ & Range (mcg/mL) \\
\hline & & Metronidazole & 0.5 & I & $\leq 0.06-1.00$ \\
\hline & & Imipenem & 0.06 & 1 & $\leq 0.03-1.00$ \\
\hline Peptostreptococcus & $10^{50}$ & TR-700 & 0.25 & 0.5 & $0.12-1.00$ \\
\hline \multirow[t]{3}{*}{ micros } & & Linezolid & I & 2 & $0.5-2.0$ \\
\hline & & Metronidazole & $\leq 0.06$ & $>32$ & $\leq 0.06$ to $>32.00$ \\
\hline & & Imipenem & $\leq 0.03$ & $\leq 0.03$ & $\leq 0.03$ to $\leq 0.06$ \\
\hline Porphyromonas & $10^{50}$ & TR-700 & 0.25 & 0.5 & $0.25-0.50$ \\
\hline \multirow[t]{3}{*}{ asaccharolytica } & & Linezolid & I & 2 & $0.5-2$ \\
\hline & & Metronidazole & I & I & $0.5-2$ \\
\hline & & Imipenem & $<0.03$ & 0.06 & $\leq 0.03$ to $\leq 0.06$ \\
\hline \multirow[t]{4}{*}{ Prevotella spp. } & $20^{50}$ & TR-700 & I & 4 & $\leq 0.06-16.00$ \\
\hline & & Linezolid & 2 & 4 & $0.25-16.00$ \\
\hline & & Metronidazole & 0.5 & $>32$ & $\leq 0.06$ to $>32.00$ \\
\hline & & Imipenem & $\leq 0.06$ & 1 & $\leq 0.03-16.00$ \\
\hline
\end{tabular}

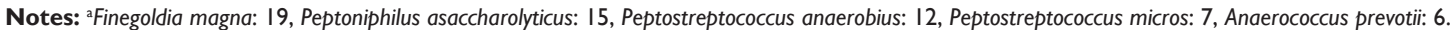
Data from Schaadt et al ${ }^{50}$ and Yum et al..$^{54}$

Abbreviations: $\mathrm{MIC}_{50}$, minimum inhibitory concentration required to inhibit the growth of $50 \%$ of organisms; $\mathrm{MIC}_{90}$, minimum inhibitory concentration required to inhibit the growth of $90 \%$ of organisms.

L3. Linezolid-selected spontaneous mutants possessed the T2500A or G2447T 23S rRNA mutations or the Gly155Arg or Gly155Arg/Met169 Leu L3 mutations.

Following 30 serial passages on antibiotic gradient plates, the MIC of tedizolid against the MSSA strain remained at $0.5 \mathrm{mcg} / \mathrm{mL}$ whereas the MIC of linezolid increased from 2 to $128 \mathrm{mcg} / \mathrm{mL}$. Reduced sensitivity to linezolid was observed after five serial passages in a medium with linezolid, associated with the Gly155Arg mutation in L3. Subsequent passages in this medium resulted in mutant strains of MSSA that had the 23S rRNA G2447T mutation alone or the G2447T mutation coupled with the Gly152Asp mutation in L3. After serial passage of the MRSA strain, tedizolid MIC values increased eightfold (from 0.25 to $2.00 \mathrm{mcg} / \mathrm{mL}$ ) while the MIC of linezolid increased 32-fold (from 1 to $32 \mathrm{mcg} / \mathrm{mL}$ ). The reduction in sensitivity of the MRSA strain to linezolid was associated initially with the Lys68Gln mutation in L4 and later by the G2576T mutation in 23S rRNA, while resistance to tedizolid was associated with a T25761C/G2576T double mutation in $23 \mathrm{~S}$ rRNA. ${ }^{64}$

A second resistance-selection study passaged eight linezolid-susceptible strains through a medium containing tedizolid. A twofold increase in the MIC of tedizolid after 14 days was found in three of the strains: MSSA, VanA-phenotype E. faecalis, and $S$. pyogenes carrying the $\operatorname{erm}(A)$ gene. All tedizolid MIC values were less than or equal to $0.5 \mathrm{mcg} / \mathrm{mL}$ at the end of the experiment and all returned to baseline following passage in nonselective media. ${ }^{53}$
According to the results of the studies described to date, tedizolid has a more favorable resistance profile than linezolid. ${ }^{53,64}$

\section{Animal studies}

Studies in murine models have evaluated antibacterial activity, pharmacokinetics, and pharmacodynamic correlates of efficacy to determine exposure-response relationships for tedizolid. A dose-range study of tedizolid phosphate in a neutropenic mouse-thigh model of MSSA and MRSA infections with linezolid as a comparator, determined that $\mathrm{AUC}_{\text {free }}$ / $\mathrm{MIC}$ is the main determinant of efficacy. ${ }^{34}$ For MSSA strains, the administration of a mean dose of tedizolid phosphate/ tedizolid of $37.7 \mathrm{mg} / \mathrm{kg} /$ day resulted in stasis and a mean dose of $66.9 \mathrm{mg} / \mathrm{kg} /$ day resulted in the reduction of $1 \mathrm{log}$ colony-forming unit (CFU)/g at 24 hours. Similarly, the administration of a mean dose of tedizolid phosphate/tedizolid of $35.3,46.6$, and $71.1 \mathrm{mg} / \mathrm{kg} /$ day resulted in stasis, reduction of $1 \log \mathrm{CFU} / \mathrm{g}$, and reduction of $2 \log \mathrm{CFU} / \mathrm{g}$ at 48 hours, respectively. Additionally, for MRSA strains, the administration of a mean dose of tedizolid phosphate of $36.2 \mathrm{mg} / \mathrm{kg} /$ day resulted in stasis and a mean dose of $58.0 \mathrm{mg} / \mathrm{kg} /$ day resulted in the reduction of $1 \log \mathrm{CFU} / \mathrm{g}$ at 24 hours. Similarly, the administration of a mean dose of tedizolid phosphate of 39.8 , 52.4 , and $105.0 \mathrm{mg} / \mathrm{kg} /$ day resulted in stasis, reduction of $1 \mathrm{log}$ $\mathrm{CFU} / \mathrm{g}$ and reduction of $2 \log \mathrm{CFU} / \mathrm{g}$ at 48 hours, respectively. There were no differences in the doses of tedizolid phosphate needed to reach these values for strains of MSSA and MRSA. When compared with linezolid, the administration of doses up to $150 \mathrm{mg} / \mathrm{kg} /$ day resulted in bacterial densities in the 
mouse thigh of approximately $1 \log \mathrm{CFU} / \mathrm{g}$ higher than the stasis values.

An in vitro study showed that tedizolid accumulated inside the macrophages and reached a ratio of approximately 10 between the intracellular and extracellular concentration. ${ }^{65}$ The value of this ratio was $1-2$ for linezolid. Based on this finding, the objective of another study was to evaluate the effect of granulocytes in the activity of tedizolid in a murine model infected with strain 33591 of MRSA compared with the same neutropenic infection model. ${ }^{66}$ It used doses of tedizolid phosphate equivalent to those humans would be given, ranging from 200 to $3200 \mathrm{mg}$ daily. The animals were evaluated at 24,48 , and 72 hours after starting treatment. In the animals with a normal immune system, bacteriostasis was obtained at an equivalent dose in humans of slightly above $100 \mathrm{mg}$ daily after 24 hours and less than $100 \mathrm{mg}$ per day at 48-hour and 72-hour endpoints. The dose equivalent to $1200 \mathrm{mg}$ given daily in humans obtained the maximum response after 24 hours and the dose equivalent to $800 \mathrm{mg}$ given daily in humans obtained the maximum response after 48 hours. Lastly, the dose equivalent to $200 \mathrm{mg}$ administered daily in humans produced a near-maximal effect after 72 hours, with no significant differences observed compared with the dose of $3200 \mathrm{mg}$ daily. In contrast, in the neutropenic animals, bacteriostasis was achieved using a dose equivalent to slightly under $2300 \mathrm{mg}$ given daily in humans after 24 hours and a dose slightly under $2000 \mathrm{mg}$ daily after 72 hours. The dose equivalent to $3200 \mathrm{mg}$ administered in humans was associated with maximum response.

Furthermore, a reduction in the colony counts of $S$. aureus strains was observed in immunocompetent mice after the administration of a dose equivalent to $200 \mathrm{mg}$ of tedizolid phosphate given in humans, whereas an increase of $1 \log \mathrm{CFU} / \mathrm{g}$ was observed in the granulocytopenic animals. This difference revealed that the antibiotic effect was mediated by the granulocytes. ${ }^{66}$

In another study, the effective dose for $50 \%$ of people (ED50) value of IV tedizolid phosphate was $2.8 \mathrm{mg} / \mathrm{kg}$ in a murine model with systemic MRSA infection and $3.3 \mathrm{mg} / \mathrm{kg}$ when this infection was caused by MSSA, while the ED50 of oral tedizolid phosphate was $3.7 \mathrm{mg} / \mathrm{kg}$ in the murine model with systemic MRSA infection and $5 \mathrm{mg} / \mathrm{kg}$ when this infection was caused by MSSA. ${ }^{31}$ These values were lower than those observed with linezolid. However, no differences were observed between the efficacy of tedizolid and linezolid in a murine model infected with five strains of $S$. aureus, four of which showed resistance to methicillin. ${ }^{67}$ Thus, exposure to tedizolid reduced the number of CFUs to $1.04-1.80 \log 10$ at
24 hours, 2.13-2.68 $\log 10$ at 48 hours, and 2.68-3.72 $\log 10$ at 72 hours, while exposure to linezolid reduced the number of CFUs to $1.36-2.02 \log 10$ at 24 hours, 2.19-3.11 $\log 10$ at 48 hours, and $2.64-3.76 \log 10$ at 72 hours. Statistically significant differences were observed in the number of CFUs for some $S$. aureus strains at 24, 48, or 72 hours, although they were not consistent throughout the study period.

Tedizolid has shown in vitro activity against strains of Nocardia brasiliensis, with $\mathrm{MIC}_{50}$ and $\mathrm{MIC}_{90}$ values of $1 \mathrm{mg} / \mathrm{L},{ }^{58}$ and both tedizolid and tedizolid phosphate have shown a high capacity for inhibiting intracellular growth of N. brasiliensis in vitro. ${ }^{68}$ Therefore, the objective of one study was to evaluate the activity of two different doses of tedizolid phosphate ( 5 or $25 \mathrm{mg} / \mathrm{kg}$ ), in monotherapy or combined with trimethoprim/sulfamethoxazole, in a murine model of actinomycosis due to $N$. brasiliensis. ${ }^{69}$ Linezolid was used as a comparator treatment and saline solution as a control. At the end of treatment, statistically significant differences were observed in the degree of infection between all groups that received the different antibiotic treatments and the control group $(P=0.004)$. However, a greater response was observed in the groups that received tedizolid phosphate at a dose of $25 \mathrm{mg} / \mathrm{kg}$, both in monotherapy and in combination with trimethoprim/sulfamethoxazole, than in the groups that received the other treatments.

Another study evaluated the activity of tedizolid phosphate at doses of $5,12.5$, and $25 \mathrm{mg} / \mathrm{kg}$ in a murine model of actinomycosis due to $N$. brasiliensis. ${ }^{70}$ Saline solution was used as a control. The animals received the treatment 1 week after infection for a period of 3 weeks. Subsequently, the treatment was suspended for 1 week and started again for another 3 weeks. At the end of treatment, statistically significant differences were observed in the degree of infection between the control group and the groups that received the three doses of tedizolid phosphate $(P<0.001)$.

One study assessed survival at 7 days in a murine model with systemic infection with penicillin-resistant S. pneumoniae after administration of tedizolid phosphate orally or intravenously at different doses. ${ }^{56}$ Four strains of penicillin-resistant S. pneumoniae were used (DR9, DR10, DR11, and DR14) and linezolid was used as a comparator treatment. The ED50 of tedizolid phosphate administered orally ranged from $5.7 \mathrm{mg} / \mathrm{kg} /$ day for the mice infected with DR9 to $11.53 \mathrm{mg} / \mathrm{kg} /$ day for those infected with DR14. This latter value was equivalent to that observed with linezolid, while the values compared with the rest of the strains were lower. Similarly, the ED50 of tedizolid phosphate administered intravenously ranged from $4.89 \mathrm{mg} / \mathrm{kg} /$ day in mice infected 
with DR9 to $10.19 \mathrm{mg} / \mathrm{kg} /$ day for those infected with DR11. These values were lower than those observed with linezolid against the four strains of penicillin-resistant S. pneumoniae.

\section{Clinical efficacy}

To date, there have been a limited number of studies in humans evaluating the efficacy of tedizolid phosphate or tedizolid in skin and soft tissue infections, and some of these are still underway. The objective of one Phase II randomized double-blind clinical trial was to evaluate the efficacy of tedizolid phosphate administered at doses of 200,300 , or $400 \mathrm{mg}$ once daily for 5-7 days in patients with complicated skin and soft tissue infections ${ }^{40}$ These included abscesses (with at least $2 \mathrm{~cm}$ of surrounding induration or requiring incision and drainage), surgical or posttraumatic wounds, and deep extensive cellulitis. A total of 192 patients were randomized between September 2008 and January 2009, of whom 188 received at least one dose of tedizolid phosphate and these 188 patients presented with a diagnosis of complicated skin and soft tissue infection (modified intent-to-treat and clinical modified intent-to-treat, respectively). Of the 188, 164 were included in the clinically evaluable population, 154 in the microbiological modified intent-to-treat population, and 133 in the microbiologically evaluable population. S. aureus strains were isolated in 139 (90.3\%) of the 154 patients in whom a baseline Gram-positive microorganism was isolated, of which $80.6 \%$ were MRSA. The clinical cure rate in clinically evaluable patients was $98.2 \%$ for the group that received $200 \mathrm{mg}$ of tedizolid phosphate and $94.4 \%$ for the group that received 300 or $400 \mathrm{mg}$ of the antibiotic; no differences were observed in terms of the type and size of the lesion or the severity of the infection. Likewise, the clinical cure rate in microbiologically evaluable patients in whom $S$. aureus strains $(\mathrm{n}=119)$ had been isolated was $96.6 \%$, reaching a value of $96.8 \%$ when the isolated strains were MRSA. Clinical failure was observed in seven patients (3.7\%).

In addition, the overall microbiological eradication rate was $97.7 \%$ in the microbiologically evaluable patients $(n=133)$, with no differences observed in terms of dose. This rate ranged from $92.6 \%$ to $100 \%$ when MRSA strains were isolated and from $88.9 \%$ to $100 \%$ when MSSA strains were isolated. Emerging pathogenic microorganisms were isolated in 2/188 (1\%) patients.

The importance of adequate dosing of tedizolid was highlighted in a study that evaluated the production of phenol-soluble modulins by $S$. aureus strains from skin and soft tissue infections at different concentrations of the antibiotic. ${ }^{71}$ Tedizolid inhibited the production of these proteins at half the MIC concentration, mainly affecting the production of phenol-soluble modulin alpha 3 and, to a lesser extent, the production of phenol-soluble modulin alpha 4. However, when the concentration of tedizolid was one-quarter and one-eighth of the MIC, it induced the production of phenol-soluble modulins, mainly in those strains with a low baseline production of phenol-soluble modulin alpha 3 . The highest increase observed in the phenol-soluble modulin alpha 3 concentration was 4.6 from $2.5 \mathrm{mcg} / \mathrm{mL}$. Similar results were obtained with the control strain, increasing the phenol-soluble modulin alpha 3 concentration from 3.90 to 5.43 and $5.63 \mathrm{mcg} / \mathrm{mL}$ with tedizolid concentrations at onequarter and one-eighth of the MIC value, respectively. This study highlights the importance of the adequate dosing of these antibiotics with an aim to minimize the potential for induction of virulence.

A Phase III randomized double-blind multicenter study was carried out to evaluate the efficacy of tedizolid phosphate $200 \mathrm{mg}$ daily taken orally for 6 days compared with linezolid $600 \mathrm{mg}$ every 12 hours taken orally for 10 days in 667 patients with acute bacterial skin and soft tissue infections from North America, South America, and Europe. ${ }^{72}$ Tedizolid was not inferior to linezolid when the primary and secondary endpoints were evaluated in the intent-to-treat analysis. ${ }^{73}$ Given that the duration of tedizolid treatment was 4 days fewer than that of linezolid, an additional analysis to the previous study was conducted in the 245 patients who were diagnosed with cellulitis with a minimum total surface area of the lesion of $75 \mathrm{~cm}^{2} .{ }^{74}$ The US Food and Drug Administration (FDA) endpoint was cessation of lesion extension and absence of fever at 48-72 hours, while the European Medicines Agency (EMA) endpoint was evaluation of the clinical result by the investigator 7-14 days after the end of treatment, both in the intent-to-treat group. The FDA endpoint was obtained in $72 \%(53 / 74)$ of the patients in the USA and in $82 \%$ (37/45) of the patients in Europe who received treatment with tedizolid, whereas, in the case of linezolid, these values were $69 \%(55 / 80)$ and $76 \%(35 / 46)$, respectively. Similarly, the EMA endpoint was obtained in $82 \%(61 / 74)$ of the patients in the USA and in $98 \%(44 / 45)$ of the patients in Europe who received treatment with tedizolid, whereas, in the case of linezolid, these values were $78 \%(62 / 80)$ and $91 \%(42 / 46)$, respectively. According to the results, the cure rate was higher in US and European patients who received tedizolid, for both the FDA and EMA endpoints.

A second Phase III randomized double-blind multicenter study is currently underway with the same objective as the 
previous study, but is evaluating the transition from IV to oral route with both tedizolid phosphate and linezolid. ${ }^{75}$

\section{Tolerability}

Based on the adverse effects described for linezolid, several studies have been conducted with tedizolid to ascertain whether this molecule improves the toxicity profile of linezolid.

In general, the tolerability of tedizolid phosphate has been evaluated in several studies. ${ }^{40,76,77}$ One of them was performed in 40 healthy subjects who received either single doses of the antibiotic ranging from 200 to $1200 \mathrm{mg}$ or placebo. ${ }^{76}$ Twenty-eight treatment-related adverse effects, all of mild severity and apparently independent of the dose, were reported, including nausea $(10 \%)$, dizziness $(6.7 \%)$, diarrhea $(6.7 \%)$, and nasal congestion $(6.7 \%)$.

In a second study with ten adolescent subjects aged between 12 and 17 years, no serious adverse effects were observed, nor were there alterations in the electrocardiogram or analytical parameters after the administration of a single dose of $200 \mathrm{mg}$ tedizolid phosphate. ${ }^{77}$ Only one patient presented with mild abdominal pain related to the tedizolid phosphate treatment.

Another study evaluated the tolerability and safety of tedizolid treatment at doses of 200,300, and $400 \mathrm{mg}$ administered during a 5-7 day period in 188 adult patients with skin and soft tissue infections. ${ }^{40}$ Adverse effects were reported in $69.1 \%$ of the patients, of which $24.6 \%$ were classified as moderate and $72.3 \%$ as mild; treatment did not have to be interrupted in any case (Table 8 ). Only $2.7 \%$ of the patients experienced serious adverse effects, none of which was attributable to the drug. None of the toxicities was dose-dependent. The adverse effects reported by the investigators as treatmentrelated included nausea $(16.5 \%)$, diarrhea $(8.5 \%$; Clostridium difficile was not isolated in any case), vomiting (6.9\%), and headache $(6.4 \%)$. One patient experienced an increase in their QT interval of more than $60 \mathrm{~ms}$.

The tolerability of tedizolid in IV infusion was evaluated in ten healthy patients who received an infusion of tedizolid phosphate $200 \mathrm{mg}$ in $250 \mathrm{~mL}$ of saline solution for 60 minutes by peripheral vein for 3 days. ${ }^{78}$ Each patient acted as their own control by receiving placebo in the other treatment arm. Phlebitis was evaluated using the Visual Infusion Phlebitis (VIP) scale and good peripheral tolerance was demonstrated.

Currently, several studies evaluating the safety profile of tedizolid are underway. One of these is a Phase II open-label multicenter clinical trial evaluating the tolerability of the administration of $200 \mathrm{mg}$ of tedizolid phosphate once daily
Table 8 Most common treatment-emergent adverse events $\left(>2 \%\right.$ of treated subjects) $(\mathrm{N}=188)^{40}$

\begin{tabular}{ll}
\hline Adverse effect(s) & $\mathbf{N}(\%)$ \\
\hline Any adverse effect & $130(69.1)$ \\
Cardiac disorders & $4(2.1)$ \\
Tachycardia & $4(2.1)$ \\
Gastrointestinal disorders & $71(37.8)$ \\
Diarrhea & $16(8.5)$ \\
Dry mouth & $4(2.1)$ \\
Nausea & $35(18.6)$ \\
Vomiting & $19(10.1)$ \\
General disorders and administration site conditions & $15(8.0)$ \\
Fatigue & $5(2.7)$ \\
Pain & $6(3.2)$ \\
Infections & $41(21.8)$ \\
Abscesses & $22(11.7)$ \\
Cellulitis & $4(2.1)$ \\
Skin infections & $8(4.3)$ \\
Investigations & $8(4.3)$ \\
Increase in blood pressure & $7(3.7)$ \\
Metabolism and nutrition disorders & $6(3.2)$ \\
Decreased appetite & $5(2.7)$ \\
Central nervous system disorders & $30(16.0)$ \\
Dizziness & $5(2.7)$ \\
Headache & $21(11.2)$ \\
Psychiatric disorders & $16(8.5)$ \\
Insomnia & $6(3.2)$ \\
Respiratory disorders & $15(8.0)$ \\
Flu & $5(2.7)$ \\
Oropharyngeal pain & $4(2.1)$ \\
Rhinorrhea & $4(2.1)$ \\
Skin and subcutaneous tissue disorders & $21(11.2)$ \\
Pruritus & $4(2.1)$ \\
Rash & $5(2.7)$ \\
Skin lesions & $7(3.7)$ \\
\hline &
\end{tabular}

in patients over 18 years of age with skin infections (major cutaneous abscesses, erysipelas, and cellulitis). ${ }^{79}$

In general, the tolerability of tedizolid phosphate is similar to that observed with linezolid, according to a Phase III study that compared the safety profile of the adverse effects of treatment with tedizolid phosphate $200 \mathrm{mg}$ once daily for 6 days or linezolid $600 \mathrm{mg}$ twice daily for 10 days in patients with skin and soft tissue infections. ${ }^{73}$ The results showed a drug-related adverse effects rate of $24.2 \%$ in the group that received tedizolid phosphate compared with $31.0 \%$ in the group that received linezolid.

One of the greatest concerns with linezolid treatment is the potential for developing blood toxicity, including anemia, thrombocytopenia, leucopenia, or pancytopenia; an association with the duration of antibiotic treatment has been observed ${ }^{80,81}$ Therefore, weekly complete blood counts are recommended, especially in patients who present with prior myelosuppression or who receive concomitant myelotoxic 
drugs, ${ }^{82}$ and treatment should be discontinued in patients who develop this adverse effect. Myelosuppression associated with linezolid is one of the main factors that limit its use.

Thus, of great therapeutic advantage would be the development of new molecules belonging to the oxazolidinone family that maintain antimicrobial activity but have a lower hematological toxicity profile. As such, the objective of several studies has been to evaluate the hematological toxicity of tedizolid phosphate. One study conducted in animals monitored signs of toxicity after the administration of increasing single doses up to $250 \mathrm{mg} / \mathrm{kg}$ IV and $2000 \mathrm{mg} / \mathrm{kg}$ orally in rats and mice of both sexes. ${ }^{83}$ The toxic effects of administration of tedizolid phosphate at doses between 10 and $100 \mathrm{mg} / \mathrm{kg}$ for 4 weeks were also evaluated. The median lethal dose ranged from 244 and $274 \mathrm{mg} / \mathrm{kg}$ for the IV route and 2000 and $2052 \mathrm{mg} / \mathrm{kg}$ for the oral route.

The repeat-dose no observed adverse effect level was $30 \mathrm{mg} / \mathrm{kg}$ for males and $10 \mathrm{mg} / \mathrm{kg}$ for females and the toxicity target organs were both the lymphatic and hematopoietic organs, such as the spinal cord, thymus, spleen, and lymph nodes.

A Phase I controlled study with linezolid $600 \mathrm{mg}$ twice daily and placebo evaluated the hematological toxicity of tedizolid phosphate at doses of 200,300 , and $400 \mathrm{mg}$ once daily administered for 21 days in 40 healthy subjects. ${ }^{84}$ No hematological adverse effects or clinically meaningful changes in blood cell counts were reported with $200 \mathrm{mg}$ oncedaily administration for 21 days. However, hematological changes increased with dose and duration of administration such that the administration of $400 \mathrm{mg}$ once daily of tedizolid phosphate resulted in hematological alterations starting during the second week of treatment. These alterations were similar to those observed with linezolid $600 \mathrm{mg}$ twice daily, and occurred at approximately the same time. In another study by the same authors, treatment discontinuation was reported in two patients who received $400 \mathrm{mg}$ of tedizolid phosphate due to a decrease in reticulocyte count in one case and in white cell count in the other, and linezolid treatment discontinuation in one patient due to a decrease in reticulocyte count..$^{85}$ Thus, only a few hematological adverse effects have been observed for tedizolid phosphate at a dose of $400 \mathrm{mg}$ used for more than 2 weeks. However, given that the clinical and microbiological efficacy is similar for the 200,300 , and $400 \mathrm{mg}$ doses,${ }^{55}$ the administration of lower doses could be considered to reduce the potential for developing myelosuppression.

Additionally, isolated cases of optic neuritis have been reported in patients treated with linezolid, who have had to discontinue treatment. ${ }^{86,87}$ Although the mechanism causing this is not entirely clear, a mechanism similar to that of optic neuropathy due to nutritional deficiency has been proposed ${ }^{88}$ Currently, a Phase I clinical trial is under way to evaluate the neurological and ophthalmologic safety of the oral administration of $200 \mathrm{mg}$ of tedizolid phosphate once daily for 10 days in healthy adults aged between 18 and 65 years. ${ }^{89}$

\section{Drug interactions}

Tedizolid does not inhibit the monoamine oxidase (MAO) system in vivo, due to greater antibiotic potency relative to MAO inhibition. This gives it an advantage over linezolid, which exercises a weak, reversible, and nonselective inhibition of MAO that can trigger potential interactions with adrenergic and serotonergic drugs. Therefore, it improves the profile of possible interactions with serotonin reuptake inhibitors and other compounds with serotonergic activity, as well as adrenergic agents, dietary tyramine, and endogenous biogenic amines with the consequent negative effects on the central nervous system and blood pressure..$^{90,91}$

Two randomized double-blind placebo-controlled crossover Phase I studies have been completed evaluating the potential for tedizolid phosphate to inhibit MAO. ${ }^{92}$ A comparison of the effects on systolic blood pressure (SBP) of the concomitant administration of tedizolid phosphate and pseudoephedrine against the administration of the antibiotic with placebo was investigated in 18 healthy subjects. No differences were observed in median maximum SBP change from baseline for pseudoephedrine treatment with tedizolid phosphate or placebo, and the number of subjects with SBP increases of $15 \mathrm{mmHg}$ or higher were similar between groups (four with tedizolid phosphate and five with placebo).

Similarly, a trial in healthy adults evaluated the dose of tyramine needed to cause a $30 \mathrm{mmHg}$ increase in SBP $\left(\mathrm{TYR}_{30}\right)$ in combination with $200 \mathrm{mg}$ tedizolid phosphate once daily or with placebo. Modest increases in sensitivity to tyramine were observed with tedizolid phosphate relative to placebo, but the $\mathrm{TYR}_{30}$ with tedizolid ( $339 \mathrm{mg}$ ) was only $28 \%$ lower that the $\mathrm{TYR}_{30}$ with placebo and was high enough to mean that adverse vascular effects due to intake of food with a high tyramine content are unlikely.

The potential for serotonin syndrome, a potentially fatal consequence of MAO inhibitor compounds, was evaluated in a mouse head-twitch model ${ }^{92}$ showing that tedizolid was comparable to placebo (vehicle) in its potential to induce head twitches, an established marker of serotonergic activity. In contrast, linezolid induced a head-twitch response comparable to fluoxetine, a compound known to increase serotonin concentrations by blocking reuptake. 


\section{Stability and compatibility}

The prodrug demonstrated water solubility greater than $50 \mathrm{mg} / \mathrm{mL}$ and excellent chemical stability, remaining unaltered after 8 hours in solutions with different $\mathrm{pH}$, including a glycosylated saline solution with a practically neutral $\mathrm{pH}$ of 6.47. ${ }^{31}$ The physicochemical properties of the prodrug resolve the water solubility problems observed during the development of other molecules, such as DA-7867, whose reduced water solubility and bioavailability by the oral administration route limited its use, despite its high antimicrobial activity. ${ }^{93}$ The compatibility of tedizolid phosphate has not been the object of any study to date; however, the dose of the drug was administered diluted in $250 \mathrm{~mL}$ of saline solution in two studies in healthy adults..$^{33,78}$

\section{Conclusion}

The emergence of ABSSSI with microorganisms resistant to current treatment options indicates a need to expand the available therapeutic arsenal. Tedizolid phosphate showed favorable results in the treatment ABSSSI in the first Phase III clinical trial. Tedizolid has more potent in vitro activity than linezolid against strains of Staphylococcus spp., Streptococcus spp., and Enterococcus spp., including strains resistant to linezolid and strains with reduced susceptibility to vancomycin or daptomycin. Moreover, tedizolid phosphate shows favorable pharmacokinetic and safety profiles that, along with a reduced potential for drug interactions, make this molecule an attractive option in circumstances in which the activity of currently available agents is limited.

\section{Disclosure}

The authors declare no conflicts of interest in this work.

\section{References}

1. Dong SL, Kelly KD, Oland RC, Holroyd BR, Rowe BH. ED management of cellulitis: a review of five urban centers. Am J Emerg Med. 2001;19(7):535-540.

2. Centers for Disease Control and Prevention (CDC). Soft tissue infections among injection drug users - San Francisco, California, 1996-2000. MMWR Morb Mortal Wkly Rep. 2001;50(19):381-384.

3. Edelsberg J, Taneja C, Zervos M, et al. Trends in US hospital admissions for skin and soft tissue infections. Emerg Infect Dis. 2009;15(9): 1516-1518.

4. Shen HN, Lu CL. Skin and soft tissue infections in hospitalized and critically ill patients: a nationwide population-based study. BMC Infect Dis. 2010;10:151.

5. Moet GJ, Jones RN, Biedenbach DJ, Stilwell MG, Fritsche TR. Contemporary causes of skin and soft tissue infections in North America, Latin America, and Europe: report from the SENTRY Antimicrobial Surveillance Program (1998-2004). Diagn Microbiol Infect Dis. 2007;57(1):7-13.

6. Moran GJ, Krishnadasan A, Gorwitz RJ, et al. Methicillin-resistant $\mathrm{S}$. aureus infections among patients in the emergency department. $\mathrm{NEngl}$ J Med. 2006;355(7):666-674.
7. Tenover FC, Goering RV. Methicillin-resistant Staphylococcus aureus strain USA300: origin and epidemiology. J Antimicrob Chemother. 2009;64(3):441-446.

8. Goering RV, Larsen AR, Skov R, Tenover FC, Anderson KL, Dunman PM. Comparative genomic analysis of European and Middle Eastern community-associated methicillin-resistant Staphylococcus aureus (CC80:ST80-IV) isolates by high-density microarray. Clin Microbiol Infect. 2009;15(8):748-755.

9. Hetem DJ, Westh H, Boye K, Jarløv JO, Bonten MJ, Bootsma MC. Nosocomial transmission of community-associated methicillin-resistant Staphylococcus aureus in Danish Hospitals. J Antimicrob Chemother. 2012;67(7):1775-1780.

10. Landrum ML, Neumann C, Cook C, et al. Epidemiology of Staphylococcus aureus blood and skin and soft tissue infections in the US military health system, 2005-2010. JAMA. 2012;308(1):50-59.

11. Hiramatsu K, Hanaki H, Ino T, Yabuta K, Oguri T, Tenover FC. Methicillin-resistant Staphylococcus aureus clinical strain with reduced vancomycin susceptibility. J Antimicrob Chemother. 1997;40(1): 135-136.

12. Smith TL, Pearson ML, Wilcox KR, et al. Emergence of vancomycin resistance in Staphylococcus aureus. Glycopeptide-Intermediate Staphylococcus aureus Working Group. N Engl J Med. 1999;340(7): 493-501.

13. Centers for Disease Control and Prevention (CDC). Staphylococcus aureus resistant to vancomycin - United States, 2002. MMWR Morb Mortal Wkly Rep. 2002;51(26):565-567.

14. Sievert DM, Rudrik JT, Patel JB, McDonald LC, Wilkins MJ, Hageman JC. Vancomycin-resistant Staphylococcus aureus in the United States, 2002-2006. Clin Infect Dis. 2008;46(5):668-674.

15. Farrell DJ, Mendes RE, Ross JE, Sader HS, Jones RN. LEADER Program results for 2009: an activity and spectrum analysis of linezolid using 6,414 clinical isolates from 56 medical centers in the United States. Antimicrob Agents Chemother. 2011;55(8):3684-3690.

16. Flamm RK, Farrell DJ, Mendes RE, Ross JE, Sader HS, Jones RN. LEADER surveillance program results for 2010: an activity and spectrum analysis of linezolid using 6801 clinical isolates from the United States (61 medical centers). Diagn Microbiol Infect Dis. 2012; 74(1):54-61.

17. Kehrenberg C, Schwarz S, Jacobsen L, Hansen LH, Vester B. A new mechanism for chloramphenicol, florfenicol and clindamycin resistance: methylation of 23S ribosomal RNA at A2503. Mol Microbiol. 2005; 57(4):1064-1073.

18. Long KS, Poehlsgaard J, Kehrenberg C, Schwarz S, Vester B. The Cfr rRNA methyltransferase confers resistance to Phenicols, Lincosamides, Oxazolidinones, Pleuromutilins, and Streptogramin A antibiotics. Antimicrob Agents Chemother. 2006;50(7):2500-2505.

19. Smith LK, Mankin AS. Transcriptional and translational control of the mlr operon, which confers resistance to seven classes of protein synthesis inhibitors. Antimicrob Agents Chemother. 2008;52(5): 1703-1712.

20. Mendes RE, Deshpande LM, Castanheira M, DiPersio J, Saubolle MA, Jones RN. First report of cfr-mediated resistance to linezolid in human staphylococcal clinical isolates recovered in the United States. Antimicrob Agents Chemother. 2008;52(6):2244-2246.

21. Toh SM, Xiong L, Arias CA, et al. Acquisition of a natural resistance gene renders a clinical strain of methicillin-resistant Staphylococcus aureus resistant to the synthetic antibiotic linezolid. Mol Microbiol. 2007;64(6):1506-1514.

22. Cui L, Tominaga E, Neoh HM, Hiramatsu K. Correlation between Reduced Daptomycin Susceptibility and Vancomycin Resistance in Vancomycin-Intermediate Staphylococcus aureus. Antimicrob Agents Chemother. 2006;50(3):1079-1082.

23. Kelley PG, Gao W, Ward PB, Howden BP. Daptomycin nonsusceptibility in vancomycin-intermediate Staphylococcus aureus (VISA) and heterogeneous-VISA (hVISA): implications for therapy after vancomycin treatment failure. J Antimicrob Chemother. 2011; 66(5):1057-1060. 
24. Miller BA, Gray A, Leblanc TW, Sexton DJ, Martin AR, Slama TG. Acute eosinophilic pneumonia secondary to daptomycin: a report of three cases. Clin Infect Dis. 2010;50(11):e63-e68.

25. Cubicin ${ }^{\circledR}$ (daptomycin for injection) for intravenous use [prescribing information]. Silver Spring, MD: Food and Drug Administration (FDA); 2012. Available from: http://www.accessdata.fda.gov/drugsatfda_docs/ label/2012/021572s039lbl.pdf. Accessed July 7, 2012.

26. Morales G, Picazo JJ, Baos E, et al. Resistance to linezolid is mediated by the cfr gene in the first report of an outbreak of linezolid-resistant Staphylococcus aureus. Clin Infect Dis. 2010;50(6): 821-825.

27. Trius Therapeutics. Tedizolid phosphate (TR-701) profile [web page on the Internet]. San Diego, CA: Trius Therapeutics; nd. Available from: http://www.triusrx.com/trius-therapeutics-tedizolid-profile.php. Accessed July 7, 2012.

28. Bae SK, Yang SH, Shin KN, Rhee JK, Yoo M, Lee MG. Pharmacokinetics of DA-7218, a new oxazolidinone, and its active metabolite, DA-7157, after intravenous and oral administration of DA-7218 and DA-7157 to rats. J Pharm Pharmacol. 2007;59(7):955-963.

29. Moellering RC. Linezolid: the first oxazolidinone antimicrobial. Ann Intern Med. 2003;138(2):135-142.

30. Colca JR, McDonald WG, Waldon DJ, et al. Cross-linking in the living cell locates the site of action of oxazolidinone antibiotics. J Biol Chem. 2003;278(24):21972-21979.

31. Im WB, Choi SH, Park JY, Finn J, Yoon SH. Discovery of torezolid as a novel 5-hydroxymethyl-oxazolidinone antibacterial agent. Eur $J$ Med Chem. 2011;46(4):1027-1039.

32. Kim E, Choi S, Im W, Rhee J. Pharmacokinetics of TR-701 (DA-7218), a new oxazolidinone in mice, rats and dogs. Poster presented at the 47th Interscience Conference on Antimicrobial Agents and Chemotherapy, September 17-20, 2007, Chicago, IL.

33. Bien P, Prokocimer P, Muñoz KA, Bethune C. Absolute bioavailability of TR-701 FA and pharmacokinetics after single and multiple dose intravenous administration in healthy adult subjects. Poster presented at the 50th Interscience Conference on Antimicrobial Agents and Chemotherapy, September 12-15, 2010, Boston, MA.

34. Louie A, Liu W, Kulawy R, Drusano GL. In vivo pharmacodynamics of torezolid phosphate (TR-701), a new oxazolidinone antibiotic, against methicillin-susceptible and methicillin-resistant Staphylococcus aureus strains in a mouse thigh infection model. Antimicrob Agents Chemother 2011;55(7):3453-3460.

35. Muñoz KA, Bien P, Prokocimer P, Bethune C. Effect of food on the pharmacokinetics of torezolid (TR-700) in healthy adult subjects. Poster presented at the 19th European Congress of Clinical Microbiology and Infectious Diseases, May 16-19, 2009, Helsinki, Finland.

36. Dreskin H, Boyea T, Barker J, Fang E, Prokocimer P. An evaluation of the absorption, metabolism, and excretion of orally administered $\left[{ }^{14} \mathrm{C}\right]-\mathrm{TR}-701 \mathrm{FA}$ in healthy subjects. Poster presented at the $51 \mathrm{st}$ Interscience Conference on Antimicrobial Agents and Chemotherapy, September 17-20, 2011, Chicago, IL.

37. Prokocimer P, Bien P, Muñoz KA, Bohn J, Wright R, Bethune C. Human pharmacokinetics of the prodrug TR-701 and TR-700, its active moiety, after multiple oral doses of TR-701, a novel oxazolidinone. Poster presented at the 48th Interscience Conference on Antimicrobial Agents and Chemotherapy, October 25-28, 2008, Washington DC.

38. Bien P, Muñoz KA, Bohn J, Wright R, Bethune C, Prokocimer P. Human pharmacokinetics of TR-700 after ascending single oral doses of the prodrug TR-701, a novel oxazolidinone antibiotic. Poster presented at the 48th Interscience Conference on Antimicrobial Agents and Chemotherapy, October 25-28, 2008, Washington DC.

39. Mondick J, Gastonguay MR, Bien P, DeAnda C, Prokocimer P. Population pharmacokinetic modeling of TR-700 in patients with complicated skin and skin structure infections. Poster presented at the 50th Interscience Conference on Antimicrobial Agents and Chemotherapy, September 12-15, 2010, Boston, MA.
40. Prokocimer P, Bien P, Surber J, et al. Phase 2, randomized, doubleblind, dose-ranging study evaluating the safety, tolerability, population pharmacokinetics, and efficacy of oral torezolid phosphate in patients with complicated skin and skin structure infections. Antimicrob Agents Chemother. 2011;55(2):583-592.

41. Housman ST, Pope JS, Russomanno J, et al. Pulmonary disposition of tedizolid following administration of once-daily oral 200-milligram tedizolid phosphate in healthy adult volunteers. Antimicrob Agents Chemother. 2012;56(5):2627-2634.

42. Sahre M, Sabarinath S, Grant M, et al. Skin and soft tissue concentrations of tedizolid (formerly torezolid), a novel oxazolidinone, following a single oral dose in healthy volunteers. Int J Antimicrob Agents. 2012; 40(1):51-54.

43. Flanagan S, Muñoz KA, Minassian S, et al. Improved pharmacokinetics of the novel oxazolidinone antibiotic tedizolid phosphate compared with linezolid in healthy subjects. Poster presented at the American College of Clinical Pharmacy (ACCP) Annual Meeting, October 20-24, 2012, Hollywood, FL.

44. Flanagan S, Morris D, Boyea T, et al. A phase 1 study of intravenously administered tedizolid phosphate in subjects with advanced renal impairment. Poster presented at the 52nd Interscience Conference on Antimicrobial Agents and Chemotherapy, September 9-12, 2012, San Francisco, CA.

45. Flanagan S, Boyea T, Dreskin H, et al. A phase 1 study of orally administered tedizolid phosphate in subjects with moderate or severe hepatic impairment. Poster presented at the 52nd Interscience Conference on Antimicrobial Agents and Chemotherapy, September 9-12, 2012, San Francisco, CA.

46. Dreskin H, Muñoz KA, Fang E, et al. Safety and pharmacokinetics of single oral administration of tedizolid phosphate in healthy elderly subjects and adult control subjects. Poster presented at the 52nd Interscience Conference on Antimicrobial Agents and Chemotherapy, September 9-12, 2012, San Francisco, CA.

47. Dreskin H, Muñoz KA, Bradley J, et al. Safety and pharmacokinetics after single oral and IV administration of tedizolid phosphate in adolescent patients. Poster presented at the 52nd Interscience Conference on Antimicrobial Agents and Chemotherapy, September 9-12, 2012, San Francisco, CA.

48. Brown SD, Traczewski MM. Comparative in vitro antimicrobial activities of torezolid (TR-700), the active moiety of a new oxazolidinone, torezolid phosphate (TR-701), determination of tentative disk diffusion interpretive criteria, and quality control ranges. Antimicrob Agents Chemother. 2010;54(5):2063-2069.

49. Betriu C, Morales G, Rodríguez-Avial I, et al. Comparative activities of TR-700 (torezolid) against staphylococcal blood isolates collected in Spain. Antimicrob Agents Chemother. 2010;54(5):2212-2215.

50. Schaadt R, Sweeney D, Shinabarger D, Zurenko G. In vitro activity of TR-700, the active ingredient of the antibacterial prodrug TR-701, a novel oxazolidinone antibacterial agent. Antimicrob Agents Chemother. 2009;53(8):3236-3239.

51. Shaw KJ, Poppe S, Schaadt R, et al. In vitro activity of TR-700, the antibacterial moiety of the prodrug TR-701, against linezolid-resistant strains. Antimicrob Agents Chemother. 2008;52(12):4442-4447.

52. Rodríguez-Avial I, Culebras E, Betriu C, Morales G, Pena I, Picazo JJ. In vitro activity of tedizolid (TR-700) against linezolid-resistant staphylococci. J Antimicrob Chemother. 2012;67(1):167-169.

53. Jones RN, Moet GJ, Sader HS, Mendes RE, Castanheira M. TR-700 in vitro activity against and resistance mutation frequencies among Grampositive pathogens. J Antimicrob Chemother. 2009;63(4):716-720.

54. Yum JH, Choi SH, Yong D, et al. Comparative in vitro activities of torezolid (DA-7157) against clinical isolates of aerobic and anaerobic bacteria in South Korea. Antimicrob Agents Chemother. 2010;54(12):5381-5386.

55. Prokocimer P, Bien P, Deanda C, Pillar CM, Bartizal K. In vitro activity and microbiological efficacy of tedizolid (TR-700) against Gram-positive clinical isolates from a phase 2 study of oral tedizolid phosphate (TR-701) in patients with complicated skin and skin structure infections. Antimicrob Agents Chemother. 2012;56(9):4608-4613. 
56. Choi S, Im W, Bartizal K. Activity of tedizolid phosphate (TR-701) in murine models of infection with penicillin-resistant and penicillinsensitive Streptococcus pneumoniae. Antimicrob Agents Chemother. 2012;56(9):4713-4717.

57. Livermore DM, Mushtaq S, Warner M, Woodford N. Activity of oxazolidinone TR-700 against linezolid-susceptible and -resistant staphylococci and enterococci. J Antimicrob Chemother. 2009;63(4): 713-715.

58. Vera-Cabrera L, Gonzalez E, Rendon A, et al. In vitro activities of DA-7157 and DA-7218 against Mycobacterium tuberculosis and Nocardia brasiliensis. Antimicrob Agents Chemother. Sep 2006;50(9): 3170-3172.

59. Gonzales RD, Schreckenberger PC, Graham MB, Kelkar S, DenBesten K, Quinn JP. Infections due to vancomycin-resistant Enterococcus faecium resistant to linezolid. Lancet. 2001;357(9263):1179.

60. Tsiodras S, Gold HS, Sakoulas G, et al. Linezolid resistance in a clinical isolate of Staphylococcus aureus. Lancet. 2001;358(9277):207-208.

61. Locke JB, Hilgers M, Shaw KJ. Mutations in ribosomal protein L3 are associated with oxazolidinone resistance in staphylococci of clinical origin. Antimicrob Agents Chemother. 2009;53(12):5275-5278.

62. Locke JB, Morales G, Hilgers M, et al. Elevated linezolid resistance in clinical cfr-positive Staphylococcus aureus isolates is associated with co-occurring mutations in ribosomal protein L3. Antimicrob Agents Chemother. 2010;54(12):5352-5355.

63. Locke JB, Finn J, Hilgers M, et al. Structure-activity relationships of diverse oxazolidinones for linezolid-resistant Staphylococcus aureus strains possessing the cfr methyltransferase gene or ribosomal mutations. Antimicrob Agents Chemother. 2010;54(12):5337-5343.

64. Locke JB, Hilgers M, Shaw KJ. Novel ribosomal mutations in Staphylococcus aureus strains identified through selection with the oxazolidinones linezolid and torezolid (TR-700). Antimicrob Agents Chemother. 2009;53(12):5265-5274.

65. Lemaire S, Van Bambeke F, Appelbaum PC, Tulkens PM. Cellular pharmacokinetics and intracellular activity of torezolid (TR-700): studies with human macrophage (THP-1) and endothelial (HUVEC) cell lines. J Antimicrob Chemother. 2009;64(5):1035-1043.

66. Drusano GL, Liu W, Kulawy R, Louie A. Impact of granulocytes on the antimicrobial effect of tedizolid in a mouse thigh infection model. Antimicrob Agents Chemother. 2011;55(11):5300-5305.

67. Keel RA, Tessier PR, Crandon JL, Nicolau DP. Comparative efficacies of human simulated exposures of tedizolid and linezolid against Staphylococcus aureus in the murine thigh infection model. Antimicrob Agents Chemother. 2012;56(8):4403-4407.

68. Vera-Cabrera L, Espinoza-González NA, Welsh O, Ocampo-Candiani J, Castro-Garza J. Activity of novel oxazolidinones against Nocardia brasiliensis growing within THP-1 macrophages. J Antimicrob Chemother. 2009;64(5):1013-1017.

69. Espinoza-González NA, Welsh O, Ocampo-Candiani J, et al. Evaluation of the Combined Therapy of DA-7218, a New Oxazolidinone, and Trimethop$\mathrm{rim} /$ Sulfamethoxazole in the Treatment of Experimental Actinomycetoma by Nocardia brasiliensis. Curr Drug Deliv. May 24, 2010.

70. Espinoza-González NA, Welsh O, de Torres NW, et al. Efficacy of DA-7218, a new oxazolidinone prodrug, in the treatment of experimental actinomycetoma produced by Nocardia brasiliensis. Molecules. 2008;13(1):31-40.

71. Yamaki J, Synold T, Wong-Beringer A. Antivirulence potential of TR-700 and clindamycin on clinical isolates of Staphylococcus aureus producing phenol-soluble modulins. Antimicrob Agents Chemother. 2011;55(9):4432-4435.

72. Trius Therapeutics. TR-701 FA vs Linezolid for the treatment of acute bacterial skin and skin structure infections (ABSSSI). In: ClinicalTrials. gov [website on the Internet]. Bethseda, MD: US National Library of Medicine; 2010 [updated November 9, 2011]. Available from: http:// clinicaltrials.gov/ct2/show/NCT01170221?term=tedizolid\&rank=4. NLM identifier: NCT01170221. Accessed July 7, 2012.
73. Trius Therapeutics. Phase 3 clinical results: TR-701 posters and publications [web page on the Internet]. San Diego, CA: Trius Therapeutics; nd. Available from: http://www.triusrx.com/trius-therapeutics-tedizolidresults.php. Accessed July 7, 2012.

74. De Anda C, Fang E, Das A, Morillo MC, Prokocimer P. Comparison of tedizolid phosphate and linezolid in investigator's assessment of clinical response in patients with cellulitis in United States and Europe from a phase 3 ABSSSI study. Poster presented at the 22nd European Congress of Clinical Microbiology and Infectious Diseases, March 31-April 3, 2012, London, UK

75. Trius Therapeutics. TR-701 FA vs linezolid for the treatment of acute bacterial skin and skin structure infections. In: ClinicalTrials. gov [website on the Internet]. Bethseda, MD: US National Library of Medicine; 2011 [updated August 1, 2012]. Available from: http:// clinicaltrials.gov/ct2/show/NCT01421511?term=tedizolid\&rank=14. NLM identifier: NCT01421511. Accessed January 10, 2013.

76. Bien P, Prokocimer P, Muñoz KA, Bohn J. The safety of single ascending oral doses of TR-701, a novel oxazolidinone prodrug antibiotic. Poster presented at the 19th European Congress of Clinical Microbiology and Infectious Diseases, May 16-19, 2009, Helsinki, Finland.

77. Bradley J, Arrieta A, Capparelli E, et al. A phase 1, open-label, multicenter, single-dose, pharmacokinetic, safety and tolerance study of oral tedizolid phosphate in 12 to 17 year old patients. Poster presented at the 22nd European Congress of Clinical Microbiology and Infectious Diseases, March 31-April 3, 2012, London, UK.

78. Muñoz KA, Bien P, Prokocimer P. Assessment of the venous tolerability of torezolid phosphate infused via a peripheral catheter: a novel approach. Poster presented at the 21st European Congress of Clinical Microbiology and Infectious Diseases, May 7-10, 2011, Milan, Italy.

79. Trius Therapeutics. A phase 2 safety and exploratory skin lesion measurement of TR-701 FA study. In: ClinicalTrials.gov [website on the Internet]. Bethseda, MD: US National Library of Medicine; 2012 [updated September 5, 2012]. Available from: http://clinicaltrials.gov/ ct2/show/NCT01519778?term=tedizolid\&rank=10. NLM identifier: NCT01519778. Accessed July 7, 2012.

80. Gerson SL, Kaplan SL, Bruss JB, et al. Hematologic effects of linezolid: summary of clinical experience. Antimicrob Agents Chemother. 2002;46(8):2723-2726

81. Birmingham MC, Rayner CR, Meagher AK, Flavin SM, Batts DH, Schentag JJ. Linezolid for the treatment of multidrug-resistant, grampositive infections: experience from a compassionate-use program. Clin Infect Dis. 2003;36(2):159-168.

82. Zyvox ${ }^{\circledR}$ (linezolid for injection, tablets, for oral suspension) [package insert]. New York, NY: Pfizer; 2012. Available from: http://www.pfizer. com/files/products/uspi_zyvox.pdf. Accessed July 7, 2012.

83. Kim D, Chung H, Choi S, Im W, Rhee J. Single and repeated-dose toxicity of TR-701 (DA-7218) in rodents. Poster presented at the 47th Interscience Conference on Antimicrobial Agents and Chemotherapy, September 17-20, 2007, Chicago, IL.

84. Prokocimer P, Bien P, MuñozA, Aster R. Hematologic effects of TR-701, linezolid and placebo administered for 21 days in healthy subjects. Poster presented at the 48th Interscience Conference on Antimicrobial Agents and Chemotherapy, October 25-28, 2008, Washington DC.

85. Bien P, Prokocimer P, Muñoz KA, Bohn J. The safety of 21-day multiple ascending oral doses of TR-701, a novel oxazolidinone prodrug antibiotic. Poster presented at the 19th European Congress of Clinical Microbiology and Infectious Diseases, May 16-19, 2009, Helsinki, Finland.

86. Javaheri M, Khurana RN, O'hearn TM, Lai MM, Sadun AA. Linezolidinduced optic neuropathy: a mitochondrial disorder? Br J Ophthalmol. 2007;91(1):111-115.

87. Frippiat F, Derue G. Causal relationship between neuropathy and prolonged linezolid use. Clin Infect Dis. 2004;39(3):439.

88. Lee E, Burger S, Shah J, et al. Linezolid-associated toxic optic neuropathy: a report of 2 cases. Clin Infect Dis. 2003;37(10):1389-1391. 
89. Trius Therapeutics. A phase 1, open-label, 10 day safety study. In: ClinicalTrials.gov [website on the Internet]. Bethseda, MD: US National Library of Medicine; 2012 [updated December 5, 2012]. Available from: http:/clinicaltrials.gov/ct2/show?term=tedizolid\&rank=11. NLM identifier: NCT01623401. Accessed July 7, 2012.

90. FDA. Zyvox (linezolid): drug safety communication: serious CNS reactions possible when given to patients taking certain psychiatric medications [web page on the Internet]. Silver Spring, MD: FDA nd [updated October 21, 2011]. Available from: http://www.fda.gov/ Safety/MedWatch/SafetyInformation/SafetyAlertsforHumanMedical Products/ucm265479.htm. Accessed July 7, 2012.
91. Shaw KJ, Barbachyn MR. The oxazolidinones: past, present, and future. Ann N Y Acad Sci. 2011;1241:48-70.

92. Flanagan S, Minassian S, Fang E, et al. Lack of MAO inhibition by tedizolid phosphate in clinical and nonclinical studies. Poster presented at the 52nd Interscience Conference on Antimicrobial Agents and Chemotherapy, September 9-12, 2012, San Francisco, CA.

93. Bae SK, Chung WS, Kim EJ, et al. Pharmacokinetics of DA-7867, a new oxazolidinone, after intravenous or oral administration to rats: intestinal first-pass effect. Antimicrob Agents Chemother. 2004;48(2):659-662.

\section{Publish your work in this journal}

Drug Design, Development and Therapy is an international, peerreviewed open-access journal that spans the spectrum of drug design and development through to clinical applications. Clinical outcomes, patient safety, and programs for the development and effective, safe, and sustained use of medicines are a feature of the journal, which has also been accepted for indexing on PubMed Central. The manuscript management system is completely online and includes a very quick and fair peer-review system, which is all easy to use. Visit http://www.dovepress.com/testimonials.php to read real quotes from published authors.

Submit your manuscript here: http://www.dovepress.com/drug-design-development-and-therapy-journal 